\title{
Labelled chemical probes for demonstrating direct target engagement in living systems
}

\author{
Hugues Prevet*,1 \& Ian Collins**,1 \\ ${ }^{1}$ Cancer Research UK Cancer Therapeutics Unit, The Institute of Cancer Research, London, SW7 3RP, UK \\ *Authors for correspondence: hugues.prevet@icr.ac.uk \\ **Author for correspondence: ian.collins@icr.ac.uk
}

Demonstrating target engagement in living systems can help drive successful drug discovery. Target engagement and occupancy studies in cells confirm direct binding of a ligand to its intended target protein and provide the binding affinity. Combined with biomarkers to measure the functional consequences of target engagement, these experiments can increase confidence in the relationship between in vitro pharmacology and observed biological effects. In this review, we focus on chemically and radioactively labelled probes as key reagents for performing such experiments. Using recent examples, we examine how the labelled probes have been employed in combination with unlabelled ligands to quantify target engagement in cells and in animals. Finally, we consider future developments of this emerging methodology.

\section{Graphical abstract:}

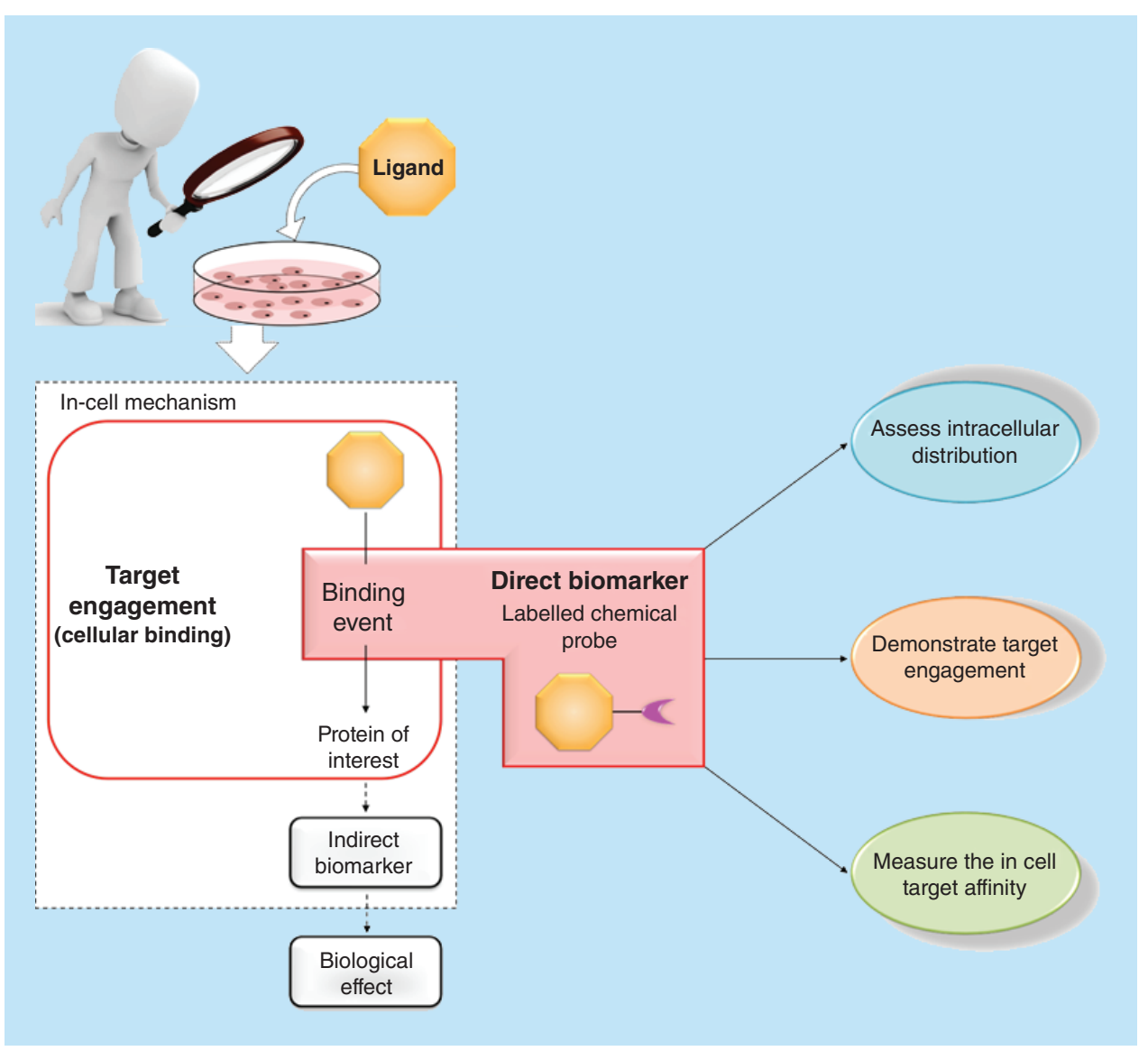

First draft submitted: 1 August 2015; Accepted for publication: 29 January 2019; Published online: 8 July 2019

Keywords: bioorthogonal reactions • fluorescent imaging • labelled chemical probes • living systems • target engagement 


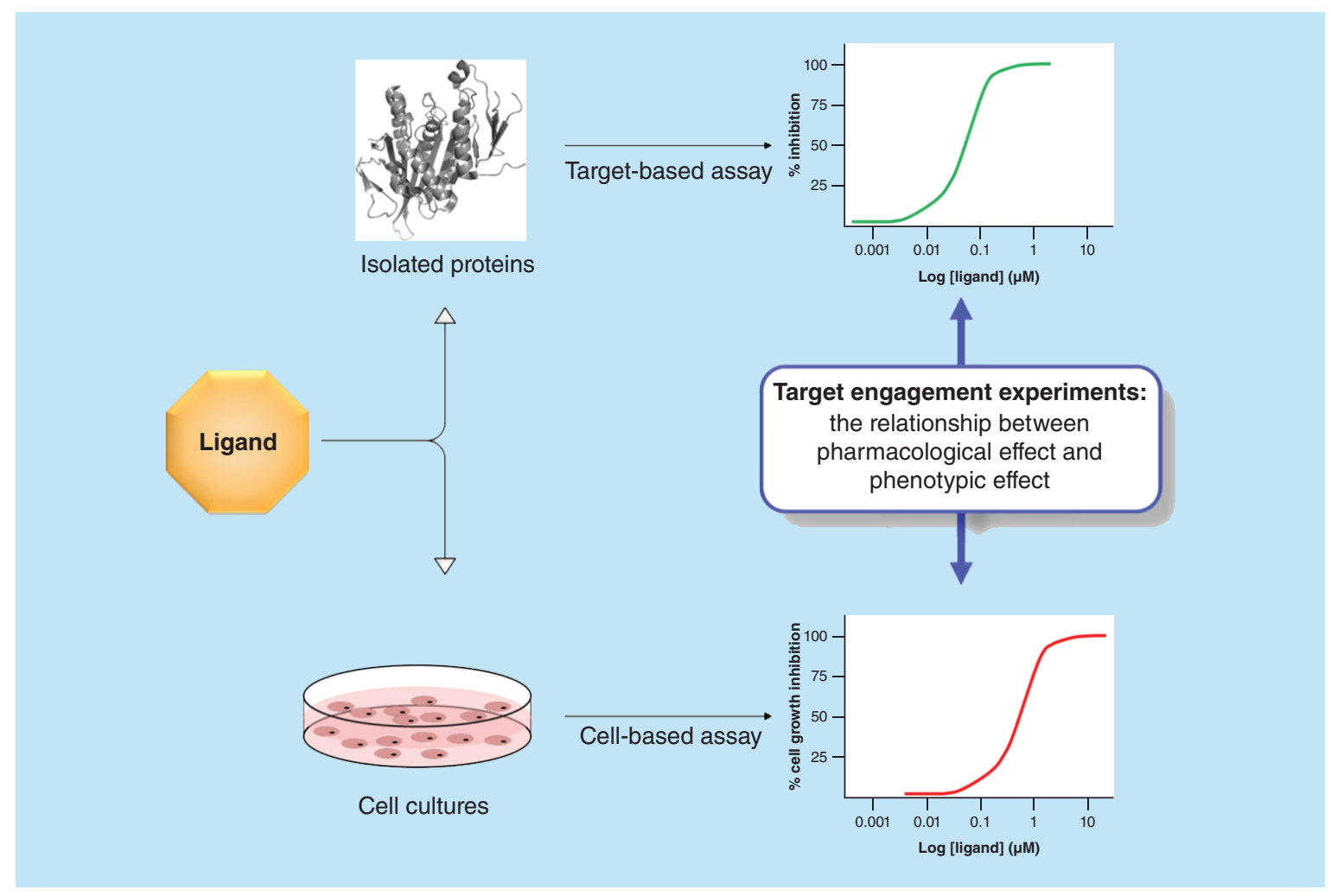

Figure 1. Target-based assay versus cell-based assay - the utility of target engagement experiments early in drug discovery.

Having the confidence that the observed biological effect is due to a ligand engaging its intended target in a relevant cellular model is one of the biggest challenges of drug discovery. Indeed, understanding target engagement has been described as one of four strategic pillars needed to drive successful drug discovery programs [1]. Monitoring target engagement is essential early in drug discovery to link target modulation with cell efficacy (Figure 1). It can also be crucial for better understanding the reasons of potential failure during later preclinical studies or even in clinical trials [2]. Drug discovery often relies on target-based assays involving isolated or purified recombinant proteins to establish structure-activity relationships (SARs) for new compounds and to optimize their potency. However, the properties of target-based in vitro assays are different to the more complex intracellular environment of cell-based assays which might result in a decrease in potency, termed 'cell drop off' [3] and potentially lead to misleading conclusions regarding the origin of the phenotype observed. For example, such differences may include changes in small molecule substrate or cofactor concentrations between in vitro assays and cells that affect ligand potency for the target (e.g., ATP competitive kinase inhibitors [4]) or the complexation of target proteins with partner biomolecules in cells that change the affinities of ligands and are not recapitulated in the in vitro assays.

Target engagement refers to the capacity of a ligand to interact with its target in living systems. It can be either demonstrated directly (assessing the binding of the ligand to its target protein) or deduced from downstream pathway effects with proximal biomarkers (e.g., assessing substrate-product changes or post-translational modifications, such as phosphorylation) [5]. However, such substrate-product transformations may be controlled by several pathways and thus changes induced by the ligand binding might not be robustly attributable to only one target [5]. This review is focused on direct target engagement experiments that provide the most immediate evidence and quantification of ligand-target association in living systems.

Direct target engagement experiments can be carried out using distinct strategies [6]. Some of these require functionalization or modification of the target protein, such as fluorescence resonance energy transfer (FRET) or bioluminescence resonance energy transfer (BRET) technologies. However, functionalization of a target protein might alter its endogenous expression and activity, and therefore the result observed in such cases might display 
reduced relevance to the unmodified system. Alternatively, the cellular thermal shift assay (CETSA) is a powerful method for monitoring the binding of unlabelled ligands to endogenous proteins in cell lysates [7]. This labelfree technology assesses the protein thermal stability following ligand binding. However, not all proteins show a detectable thermal stabilization upon ligand binding [8]. Hence, new methods have recently emerged to demonstrate direct target engagement of unlabelled ligands with endogenous proteins at cellular or subcellular resolution. These methods rely on functionalized reporter ligands that, for convenience, we refer to here as labelled chemical probes. Using selected examples from recent literature, we will examine how labelled chemical probes have been used to demonstrate direct target engagement in living systems. Most examples described to date involve cell lines, but increasingly direct target engagement in animal models has been explored, and PET imaging of target engagement in tissues using radiolabelled chemical probes is clinically applicable.

\section{The distinction between pharmacological tools \& labelled chemical probes Pharmacological tools}

Although both pharmacological tools and labelled chemical probes have a major place in drug discovery, there are differences regarding their design and applicability. Pharmacological tools are of particular importance in the chemical biology field. They are typically defined as small molecules able to modulate a specific protein's function in order to interrogate the role(s) of the targeted proteins in living systems such as cells, tissues and animals. Complementary to genetic tools such as RNAi and CRISPR, pharmacological tools can be used for elucidating the biological mechanisms leading to a specific phenotypic effect and therefore are key reagents for target validation in drug discovery [9-11]. Typically, pharmacological tools have to be comprehensively optimized and characterized to avoid generating misleading results when they are used as specific reagents to perturb biological systems [10,11]. Data sources, such as The Chemical Probes Portal [12] and Probe Miner [13], have been established to support the selection of high quality of pharmacological tools. The current consensus for developing highquality pharmacological tools places an emphasis on potency, selectivity, broad profiling and careful controls [12]. Interestingly, proof-of-target engagement in a relevant cellular model is one requisite for validating a small-molecule as a high-quality pharmacological tool [12]. This specific requisite can be assessed using labelled chemical probes.

\section{Labelled chemical probes}

Labelled chemical probes have recently emerged as a powerful tool to investigate and characterize ligand-protein interactions in cellular models. In contrast to pharmacological tools, less stringent properties are required for labelled chemical probes. However, such probes are still required to be suitably potent and selective, and these bioactive molecules additionally must bear ligation handles to perform bioorthogonal reactions with appropriate reactive-reporter partners in order to detect the probe-protein complex formed in cells [14].

Bioorthogonal reactions are particularly attractive in chemical proteomic studies because they can take place in cellular systems without interfering with biological processes [15]. These reactions have to be fast, compatible under physiological and aqueous conditions, and highly selective [16]. It is beyond the scope of this review to give a catalog and description of all existing bioorthogonal reactions, which have already been extensively reviewed [17-19]. In addition to the existing bioorthogonal reactions, guidelines to construct new bioorthogonal reagents have been suggested to extend the bioorthogonal toolbox [20,21]. Among 20 unique reactions compliant with the features listed above, two have received considerable interest: the Strain-promotedazide-alkyne cycloaddition (SPAAC) and the inverse-electron demand Diels-Alder (IEDDA) reaction (Figure 2) [22]. The SPAAC is a copper-free variant of the azide-alkyne cycloaddition which is less toxic and therefore more compatible with biological environments, whereas the IEDDA is a reaction between a strained dienophile and a diene that is sufficiently reactive to proceed at physiological temperature and pressure conditions.

The detection of the probe-protein complex can be carried out using different reactive-reporter partners such as affinity reporters or imaging reporters. Reactive-biotin partners or affinity reporters are molecules where the bioorthogonal moiety is attached to biotin. Biotin binds to streptavidin and avidin with an extremely high affinity allowing enrichment and isolation of the probepro-protein complexes by pulldown experiments [23]. The probe-protein complex can be then detected by immunoblot analysis. However, such affinity reporters are not cellpermeable due to their large size, which precludes performing the detection step in living cells. Instead, detection is usually performed on cell lysates or fixed cells.

Alternatively, reactive-fluorescent partners or imaging reporters are molecules where the bioorthogonal moiety is linked to a fluorophore. The probe-protein complex can be visualized by in-gel fluorescence analysis and/or 


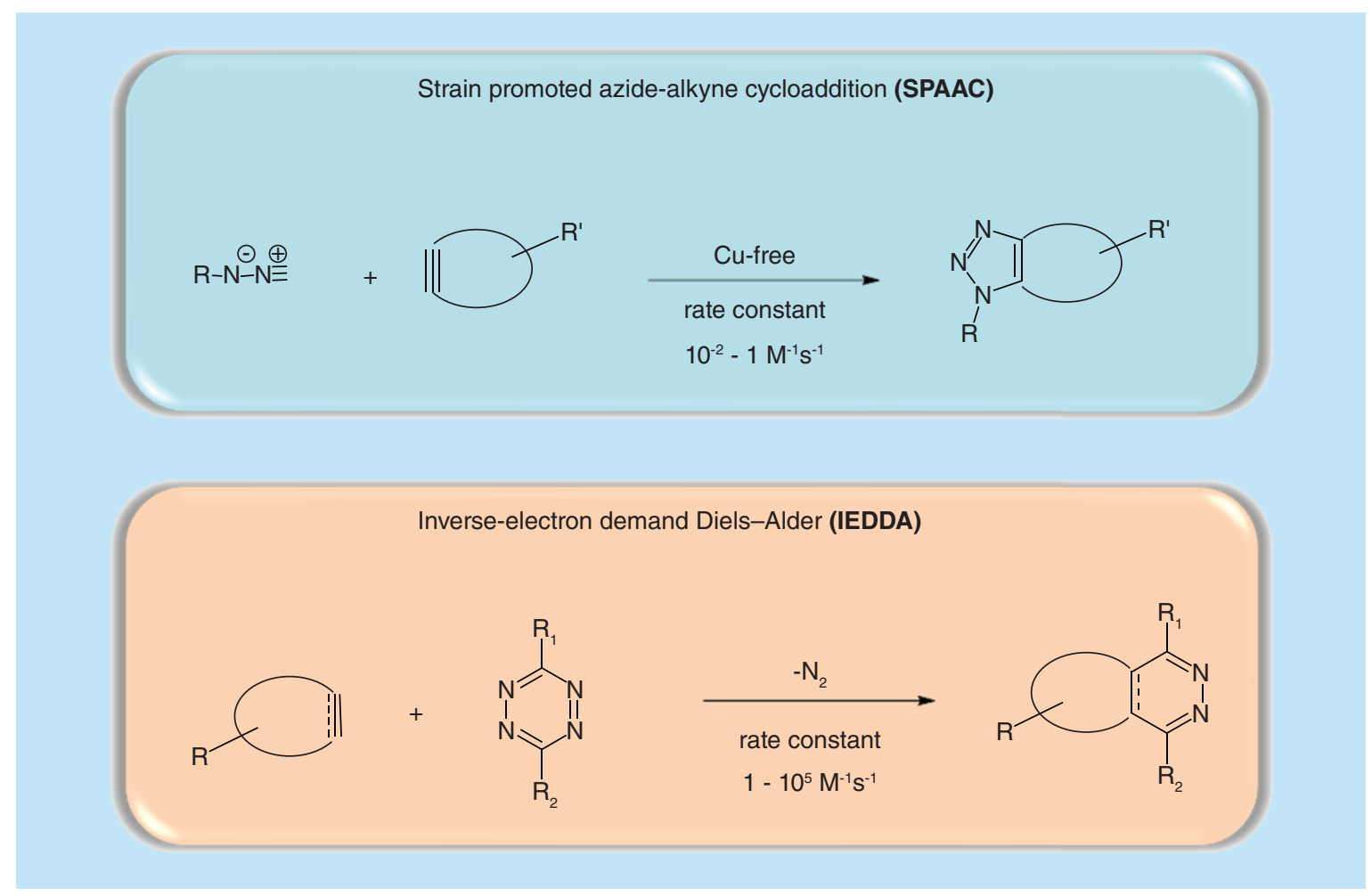

Figure 2. Strain-promoted azide-alkyne cycloaddition and inverse-electron demand Diels-Alder bioorthogonal reactions.

by confocal microscopy. Common fluorophores employed are cyanine, rhodamine, coumarin and BODIPY dyes. In contrast to the affinity reporters, many of the imaging reporters are cell permeable, and therefore they can be applied in live cells.

A promising development of tetrazine-based imaging reporters uses the fluorescence quenching effect of tetrazine [24]. Interestingly, the tetrazine moiety which is incorporated to perform the bioorthogonal reaction is also able to quench the fluorescence of the fluorophore. Therefore, such fluorogenic turn-on reporters increase fluorescence upon the bioorthogonal reaction and destruction of the tetrazine functional group. This feature appears to be very attractive for in cell imaging, as it reduces the background fluorescence and avoids stringent washing cycles, which risk depleting the amount of ligand-target complex when binding of the labelled chemical probe is reversible. The first generation of fluorogenic reporters (tetrazine-BODIPY with flexible linkers) showed a moderate turn-on ratio of around 10- to 20-fold after the IEDDA reaction. By exploiting through-bond energy transfer with modified linkers, new generations of tetrazine-BODIPY and tetrazine-coumarin have been developed exhibiting 1000- to 10,000-fold fluorescence enhancement after the bioorthogonal reaction [25,26]. Finally, a set of novel fluorogenic tetrazine-based imaging reporters have been synthesized to cover the entire emission range from green to far-red [27].

Table 1 lists the two types of labelled chemical probe/reactive-reporter partner systems that have been exploited to carry out target engagement experiments in living systems. They are sorted from major to minor changes regarding the structure of the bioactive molecule.

As described in Table 1, the ligand can be functionalized with:

- A photoreactive and a bioorthogonal group. The photoreactive group serves to covalently attached the chemical probe to the protein upon UV irradiation [28];

- A bioorthogonal group.

In both cases, the reactive-reporter partner employed for detecting and analyzing the probe-protein complex is an imaging reporter and/or an affinity reporter. 


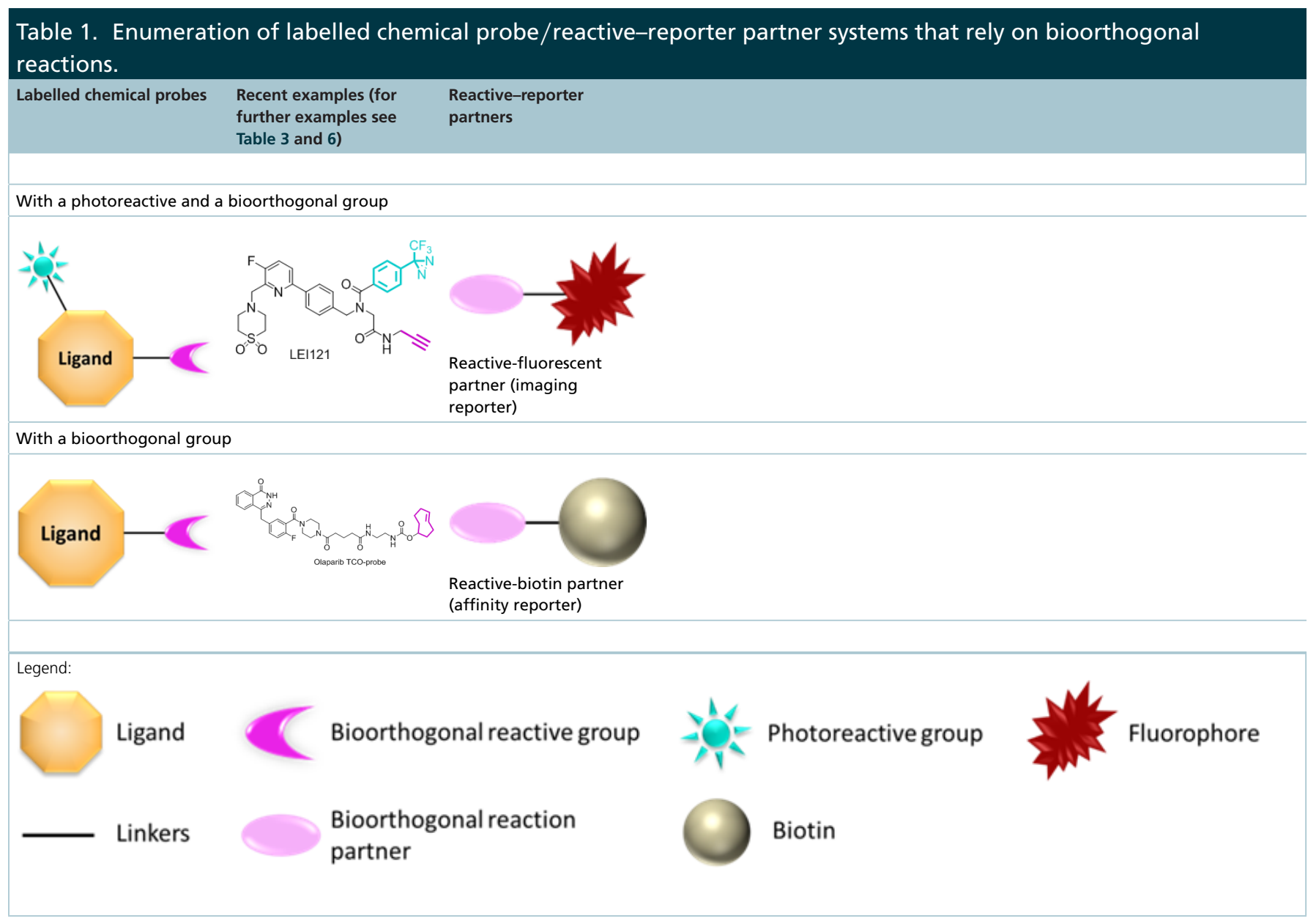

Not all target engagement experiments involve the use of bioorthogonal reactions. Indeed, the use of a labelled chemical probe where the ligand is functionalized with a cell-permeable dye to determine target engagement in vitro and in vivo has been recently described [29]. Similarly, another bioorthogonal reaction-free system consists of incorporating a radiolabelled atom into the structure of the ligand for performing positron emission tomography (PET). This approach has been applied preclinically in vivo and translated into the clinic. These two labelled chemical probe strategies are summarized in Table 2.

The systems described in Table 1 and Table 2 have been used to demonstrate that the labelled chemical probe engages its intended target in living systems. However, labeling a bioactive molecule can change its physicochemical properties, and the resulting effect may be different to the unlabelled ligand. Hence, competition assays using unlabelled ligands in combination with labelled chemical probes have been designed to assess and quantify target occupancy across series of related compounds. Such assays depend on the biochemical competition between the unlabelled ligand and the probe. Therefore, the labelled chemical probe reports only on occupancy at a specific binding site or binding sites that are allosterically coupled to it. Target occupancy refers to the measurement of protein directly bound by an unlabelled ligand. In the following section, we use selected examples from recent literature to show how labelled chemical probes in conjunction with unlabelled ligands are used to demonstrate target engagement in living systems and assess target occupancy.

\section{Target occupancy in cells with labelled chemical probes bearing photoreactive-bioorthogonal groups}

The first approach described here consists of functionalizing a ligand with a photoreactive and a bioorthogonal group. Photoreactive groups have been largely used for photoaffinity labeling (PAL) strategies to identify interactions between bioactive molecules and proteins in cells [30]. The PAL strategies and the chemistry of photoreactive groups have been extensively reviewed [28,30,31]. In short, upon photoactivation, these groups are converted into highly 
Table 2. Enumeration of bioorthogonal reaction-free systems based on two distinct labelled chemical probes.

With a cell-permeable dye

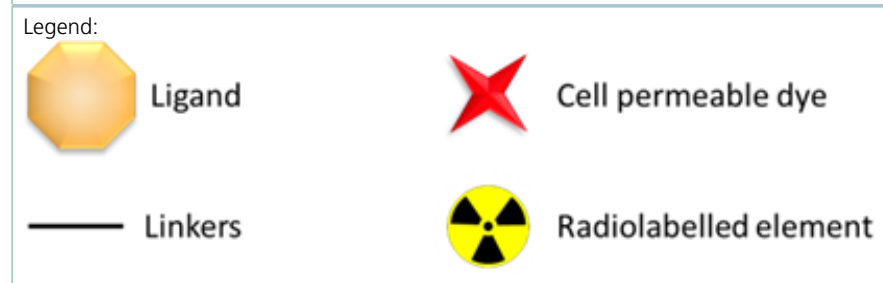

reactive intermediates that can covalently bond to the nearest neighbor molecule, which ideally is the target protein of the functionalized probe. Benzophenones, aryl azides and alkyl diazirines have been widely employed as photoreactive groups.

\section{Design of chemical probes for PAL}

Once a suitably potent, cell-active and selective ligand has been selected, the sites of functionalization have to be chosen carefully not to disturb the binding of the resulting labelled chemical probe to the protein. For that purpose, the bioorthogonal group is commonly placed in the solvent exposed region of the ligand. Standard medicinal chemistry structure-activity relationships (SARs), crystal structures of ligands bound to target proteins or NMR structural studies are useful to identify the solvent exposed regions of particular bound ligands. Conversely, due to its photocrosslinker role, the photoreactive group has to be adequately located to covalently link the probe with its binding protein without impairing the probe-protein interaction. Once synthesized, the in vitro potency as well as the cell activity of the probes are typically measured on target- and cell-based assays. In parallel, the efficiency of the bioorthogonal reaction can be confirmed in vitro (e.g., by LC-MS monitoring of reaction progression) to inform on suitable incubation conditions for the target engagement experiments.

\section{General workflow for measuring target occupancy in cells with PAL probes}

The general workflow for measuring target occupancy in cells with PAL probes is shown in Figure 3Figure 2. Cells are treated with increasing concentrations of the unlabelled ligand of interest followed by incubation with the PAL probe at one concentration. After UV-irradiation to crosslink the bound probe to its target partner, cells are lysed and bioorthogonal reaction with a reactive-reporter partner is performed. An imaging reporter can be used for visualization by SDS-PAGE and in-gel fluorescence (upper panel; Figure 3). An affinity reporter can also be used for target enrichment by pulldown with streptavidin followed by analysis by immunoblot (lower panel; Figure 3Figure 2). In either case, quantification of the fluorescent band corresponding to the labelled protein of interest reveals a dose-dependent decrease in photolabeling with increasing concentration of the unlabelled ligand due to competition for the target binding sites, allowing the in-cell affinity of the ligand to be derived. 


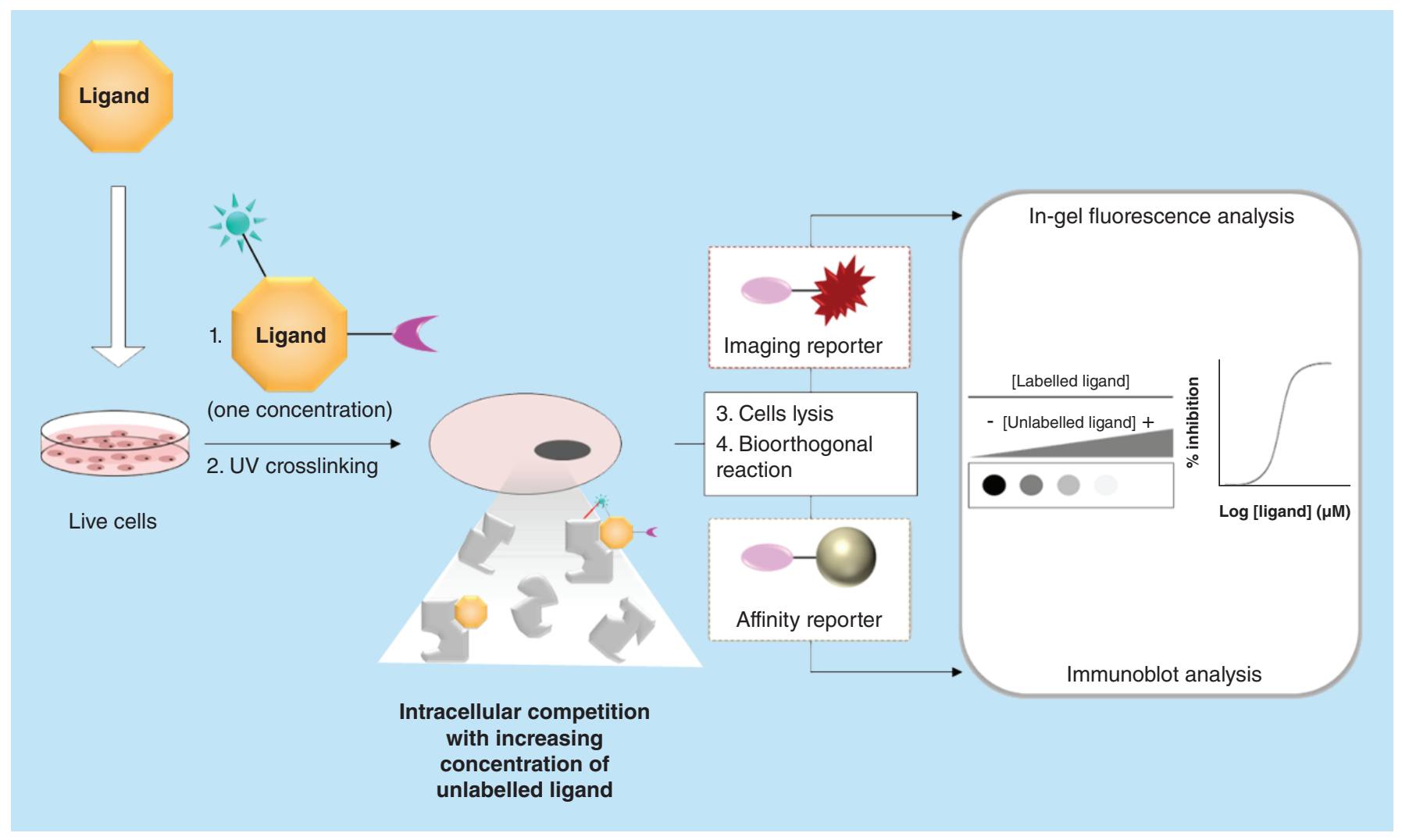

Figure 3. General workflow for measuring target occupancy in cells through labelled chemical probes bearing photoreactive and bioorthogonal groups.

\section{Recent examples}

Table 3 highlights three examples where PAL chemical probes have been used to measure the occupancy of three distinct targets. The PDE10A is a potential target for the treatment of Parkinson and Huntington diseases. The clinical candidate MP-10 was used as starting point for developing a target occupancy assay [32]. Based on the structure of the catalytic domain of PDE10A, positions were identified for the attachment of benzophenone photoreactive group and an alkyne bioorthogonal group without impairing the potency and selectivity of the resulting labelled chemical probe, PF-06481942. The potency of PF-06481942 was assessed in a phosphodiesterase assay using recombinant enzyme, showing it potently inhibited PDE10A $\left(\mathrm{IC}_{50}=29 \mathrm{nM}\right)$, comparable with the parent inhibitor MP-10 $\left(\mathrm{IC}_{50}=1.26 \mathrm{nM}\right)$. After establishing the optimal probe concentration $(30 \mathrm{nM})$ and UV-irradiation time $(20 \mathrm{~min})$ for PDE10A labeling with PF-06481942, the potency and selectivity of MP-10 for PDE10A in tissue lysates was determined using a competition assay format (MP-10 concentration range: 0-3 $\mu \mathrm{M}-$ $\mathrm{IC}_{50}=4.2 \mathrm{nM}$ ). Encouraged by this promising result, the same approach was applied to cell cultures, showing that MP-10 engaged its target PDE10A with cellular $\mathrm{IC}_{50}=1.7 \mu \mathrm{M}$. In the cell culture assay, a higher concentration of PDE10A was used $(1 \mu \mathrm{M})$ in combination with different concentrations of MP-10 $(0-30 \mu \mathrm{M})$. Interestingly, this study also highlighted how the PAL chemical probe revealed that the binding profile of a bioactive molecule in native tissues differed from the profile observed using recombinant proteins.

In another example [33], a PAL chemical probe for analyzing cellular PS1-NTF was developed from the ligand BMS-163. A set of probes was prepared by modifying the photoreactive group and the position of the bioorthogonal group. A benzophenone-containing probe was able to covalently bind PS1-NTF, whereas a diazirine-containing probe was not. It was proposed that the reactive carbene generated from the diazirine could be solvent exposed and therefore quenched before labeling the protein. This study also illustrated the influence of the electronic effect of the benzophenone substituents for the photo-crosslinking step. For example, the probe functionalized with a para-nitro benzophenone labelled many off-target proteins which were not competed by the ligand BMS-163. In contrast, the probe functionalized with para-methoxy benzophenone, having a similar potency in a biochemical 
able 3. Recent examples of target occupancy using labelled chemical probes bearing photoreactive and bioorthogonal groups.

Target

Inhibitor labelled chemical probe reactive-reporter partner

PDE10A

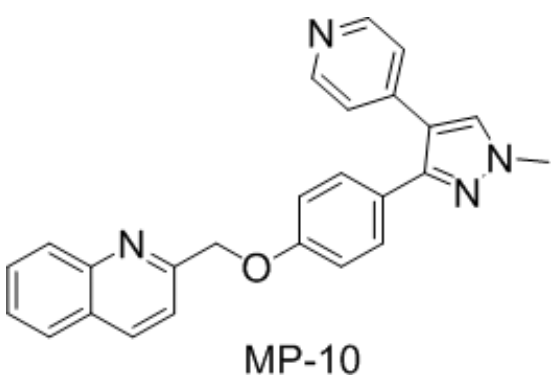

Target occupancy assessment

- Competition binding assay with MP-10

- Fluorescence-based analysis with TAMRA-azide

- Quantification of the fluorescent band of PDE10A leads to in-cell binding affinity

$\mathrm{IC}_{50}=1.7 \mu \mathrm{M}$

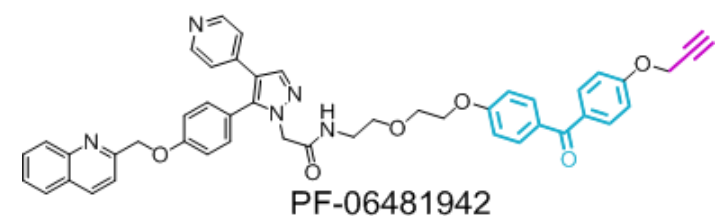

\section{ii}

TAMRA-azide for in gel fluorescence analysis<smiles>CN(C)c1ccc2c(-c3ccc(C(=O)NCCCN)cc3C(=O)[O-])c3ccc(=[N+](C)C)cc-3oc2c1</smiles>

TAMRA-azide

PS1-NTF<smiles>NC(=O)C(CCC(F)(F)F)N(Cc1ccc(-c2ncon2)cc1F)S(=O)(=O)c1ccc(Cl)cc1</smiles>

- Competition-binding assay with BMS-163

- Immunoblot-based analysis with biotin-azide

- Quantification of the fluorescent band of PS1-NTF leads to in-cell binding affinity

$\mathrm{EC}_{50}=16 \mathrm{nM}$

BMS-163 
Table 3. Recent examples of target occupancy using labelled chemical probes bearing photoreactive and bioorthogonal groups. (cont.).

Target
Ref.

(B)<smiles>C#CCNC(=O)CN(Cc1ccc(-c2ccc(F)c(CN3CCS(=O)(=O)CC3)n2)cc1)C(=O)c1ccc(C(N)(N)C(F)(F)F)cc1</smiles>

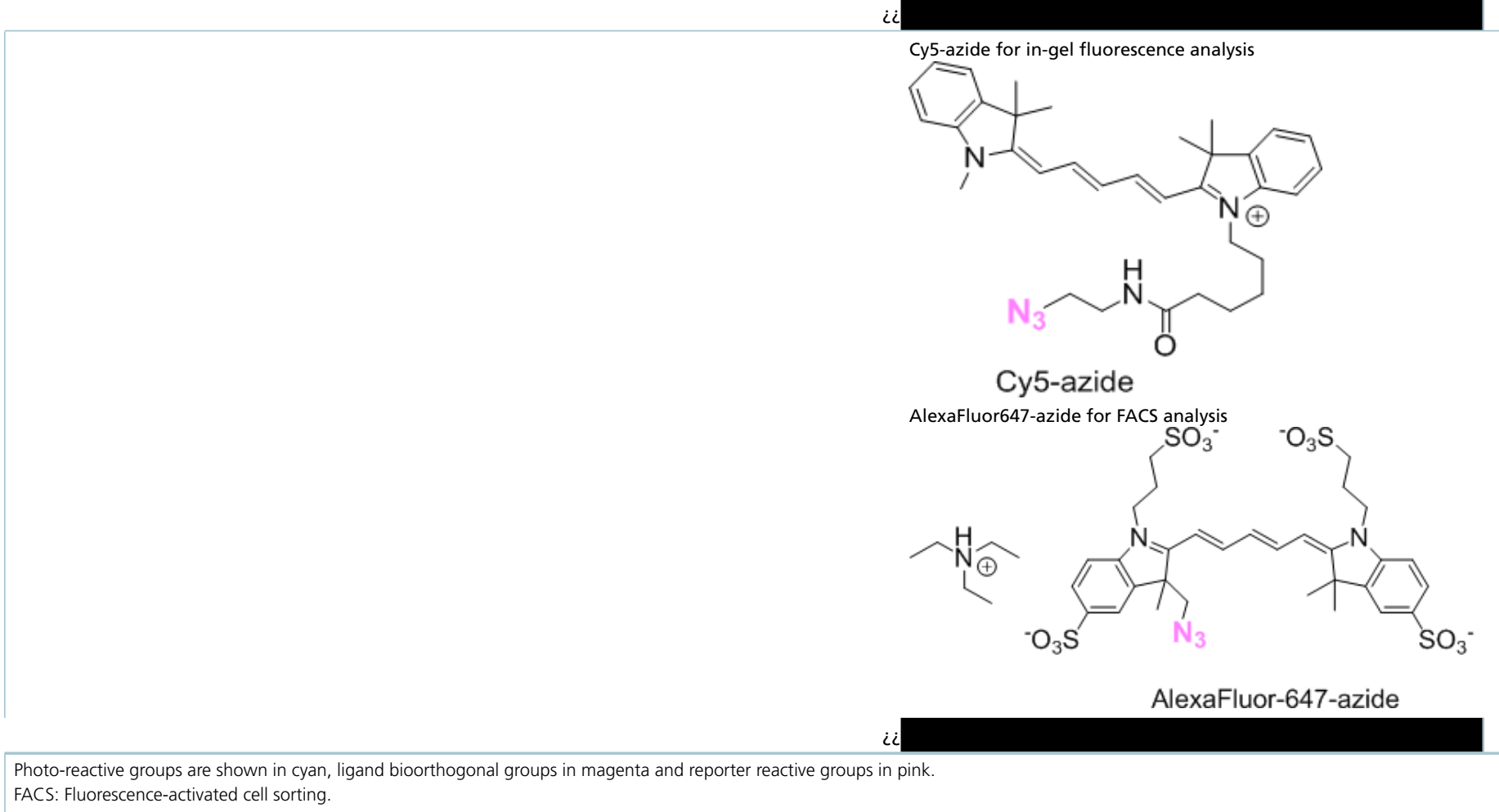


assay, showed more specific labeling. This suggests a potential difference in the reactivity of the two carbenes generated after UV-irradiation leading to differences in the proteins labelled. Placement of the alkyne within the probe had a significant impact on the magnitude of the labeling but without changing the in vitro potency. This was rationalized as due to the different placement of the alkyne group inducing slightly different binding modes of the probe, resulting in distinct alignments of the benzophenone group within the protein and therefore modifying the labeling efficiency of the photoreactive group. Moreover, this location change could also influence the efficiency of the bioorthogonal reaction. The optimized 163-BP3 probe was used at one concentration $(20 \mathrm{nM})$ to measure target occupancy in cultured primary cortical neurons and a dose-dependent decrease in PS1-NTF labeling was observed with increasing concentrations of BMS-163 $\left(0-3 \mu \mathrm{M}-\right.$ cell EC $\left.\mathrm{E}_{50}=16 \mathrm{nM}\right)$.

In the last of the selected examples, a PAL chemical probe was prepared to investigate the $\mathrm{CB}_{2} \mathrm{R}$ [34]. The ligand LEI101 with selectivity, high solubility and oral efficacy in a clinically relevant murine model was chosen as the starting point. After identifying the sites of functionalization based on standard SAR analysis and a docking study, the probe LEI121 was synthesized and showed similar potency to the ligand LEI101. Membrane preparations from $\mathrm{hCB}_{2} \mathrm{R}$-overexpressing $\mathrm{CHO}$ cells were treated with $2 \mu \mathrm{M}$ of LEI121, and two orthogonal reactive-reporter partners were used to demonstrate target engagement of a set of inhibitors tested at one concentration $(20 \mu \mathrm{M})$. An azide-biotin partner for affinity enrichment by pull-down experiment with avidin enabled receptor identification by mass-spectrometry based analysis, whereas an azide-fluorophore partner allowed identification of the interaction between the probe and protein by in-gel fluorescence. This analysis led to the detection of two forms of glycosylation of $\mathrm{CB}_{2} \mathrm{R}$. Using a competition assay format and the imaging reporter, target engagement of several ligands with distinct scaffolds was assessed in a concentration range $(0-10 \mu \mathrm{M})$. All compounds displayed a concentrationdependent displacement of the probe LE121. Interestingly, the strongest diminution in $\mathrm{CB}_{2} \mathrm{R}$ labeling was observed for the ligand with the lowest $\mathrm{IC}_{50}$ in the biochemical assay. The endogenous $\mathrm{CB}_{2} \mathrm{R}$ engagement by these ligands was studied in human cells using fluorescence-activated cell sorting (FACS) to identify labelled cells. Cells treated with $10 \mu \mathrm{M}$ of LEI121 gave a population of cells showing an increase in fluorescence which was significantly reduced when the cells were preincubated with different concentrations of unlabelled ligands $(10-20 \mu \mathrm{M})$.

Although it is beyond the scope of this review, it is notable that photoreactivity has been successfully coupled with fragment-based drug discovery to carry out the chemical proteomic analysis of thousands of reversible fragment-protein interactions in human cells and characterize the interactions, allowing further optimization of these fragments into selective ligands [35]. In the same way, photoreactive groups have also been used to identify new drug targets as well as to map the ligand binding sites of several proteins [36,37].

In summary, these PAL chemical probes have been applied with clear success to demonstrate direct target engagement and assess target occupancy in cells. The covalent attachment of the probe to the target protein after UV-irradiation avoids disruption of the interaction during experimental manipulations [32,33] offering a clear advantage over reversible labeling strategies, described in the following sections. However, the use and manipulation of labelled chemical probes bearing photoreactive groups can be restricted compare with the other probes. Indeed, due to their chemical and UV-sensitivity, it is not always straightforward to install these groups during synthesis. Another consideration is the possibility of cell toxicity when longer UV-irradiation times are applied to maximize the cross-linking in cells [32].

\section{Target occupancy in cells with labelled chemical probes bearing a bioorthogonal reactive group}

The second approach focuses on the use of labelled chemical probes only functionalized with a bioorthogonal reactive group. Recently, a covalent inhibitor tagged with a bioorthogonal reactive group has been employed to visualize the protein ERK1/2 in cells [38] and to study the selectivity of the untagged covalent inhibitor in cells [39]. Although it is beyond the scope of this review to comprehensively cover covalent inhibitors, it is worth mentioning that such bioorthogonal covalent probes provide a unique opportunity to identify potential cellular off-targets of the unlabelled covalent inhibitors that could lead to toxicity [40].

As mentioned previously, the ligand selected as starting point to develop such probes has to be suitably potent and cell active with favorable physicochemical properties. Then, the bioorthogonal reactive group has to be positioned appropriately to minimally disrupt the probe's binding at the target. Subsequently, the efficiency of the bioorthogonal reaction can be assessed using distinct bioorthogonal partners. Once synthesized and assessed in target- and cell-based assays to confirm that the probes are biologically equivalent to their parent molecules, the probes can be employed to carry out probe-target colocalization studies in cells. 


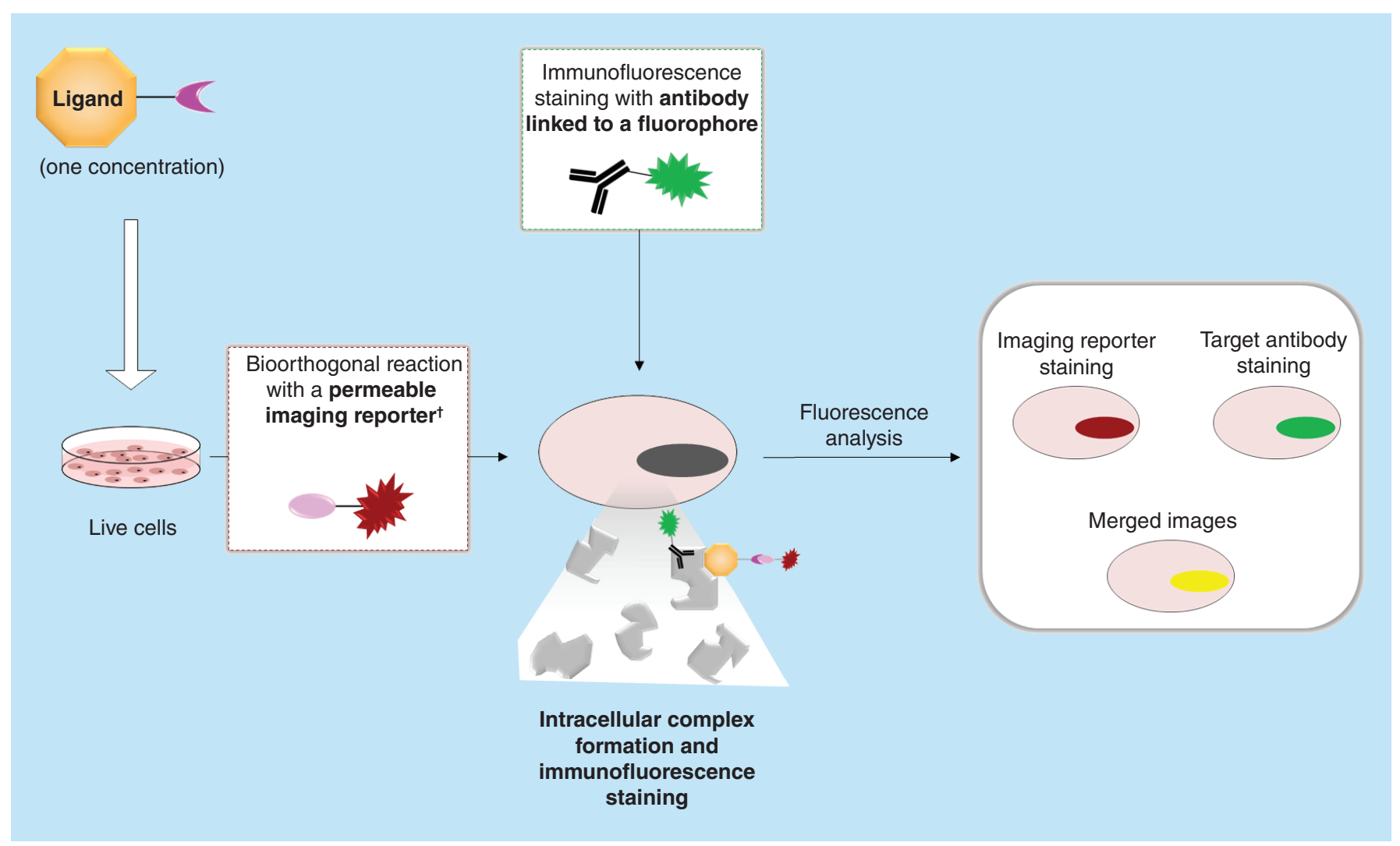

Figure 4. General workflow for assessing probe-target colocalization in cells.

$\dagger$ If the imaging reporter is notcell permeable, cell fixationand permeabilisation steps arerequired.

\section{Probe-target colocalization in cells with labelled chemical probes bearing a bioorthogonal reactive group}

The workflow to perform a probe-target colocalization study is described in Figure 4. Cells are treated with the labelled chemical probe at one concentration and incubated with a permeable imaging reporter for performing the bioorthogonal reaction inside living cells. If the imaging reporter is not cell permeable, fixation and permeabilization of the cells is required before the detection step. Immunofluorescence antibody staining of the protein of interest and imaging of the probe-reporter conjugate are enabled by confocal microscopy, allowing assessment of the subcellular distributions of the labelled chemical probe and the target protein, respectively. The merged images highlight the colocalization of the probe-target complex in cells (Figure 4).

As an example, one study [41] developed probes for imaging inhibitor binding to the DNA damage repair protein PARP1 in cells. The drug molecule olaparib was selected as the starting point and a set of probes bearing different bioorthogonal groups was synthesized, all of which retained similar potency to olaparib (Table 4). The reactivity of several reactive-reporter partners in comparison with nonreactive control compounds was investigated because side reactions might happen between the reactive-reporter partners and cellular components leading to ambiguous results. No covalent protein binding was observed for most of the reactive-reporter partners used, confirming the biorthogonality of the ligation chemistry.

Each probe was assessed at concentration $(2 \mu \mathrm{M})$ in confocal experiments using the appropriate imaging reporter for visualizing subcellular location. In order to avoid washing out the probe when using a cell-permeable imaging reporter, which can require stringent washing procedures to lower the unspecific background staining, the authors chose to fix and permeabilize the cells before adding the imaging reporter. Although all three probes studied retained potency in a biochemical PARP1 assay, the use of either SPAAC or CuAAC bioorthogonal reactions (systems 2 and 3 , respectively; Table 4) led to unspecific staining of entire cells when compared with cells treated with DMSO. This unspecific staining was likely due to the low efficiency of the bioorthogonal reactions and/or unspecific interactions between the imaging reporter and cell components. Interestingly, when the IEDDA reaction was used, a specific 

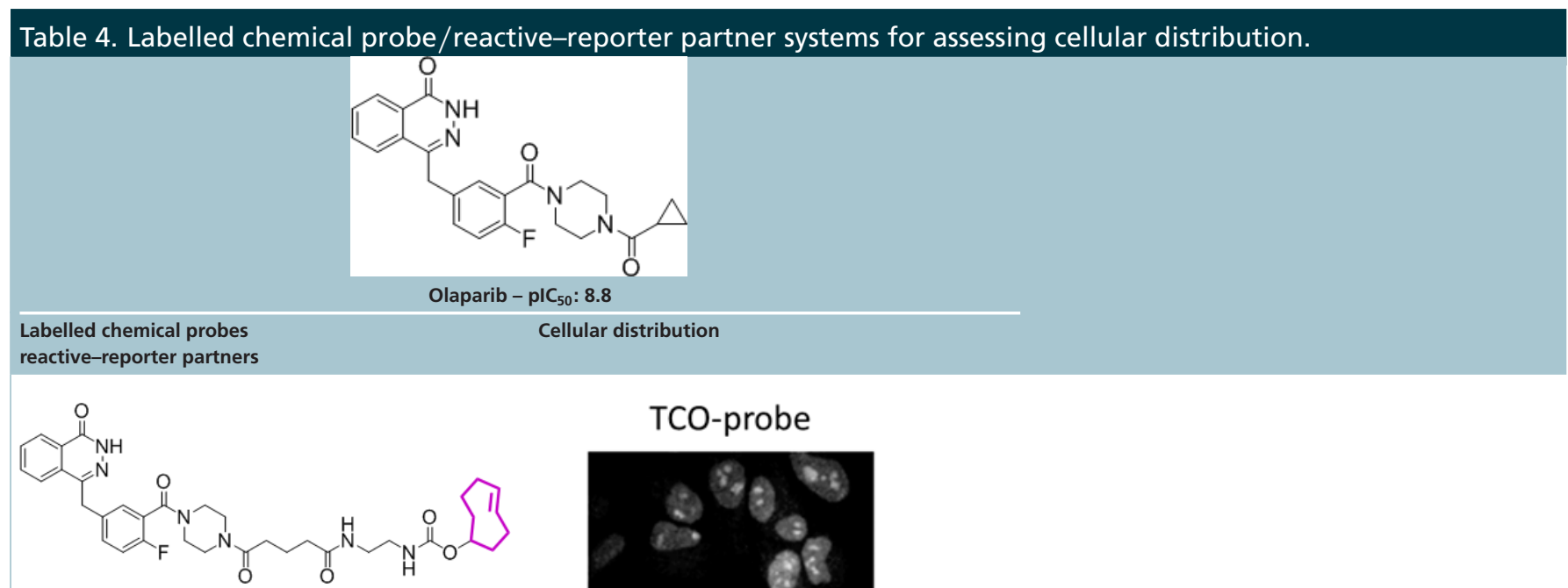

TCO-probe - pIC $_{50}: 7.5$
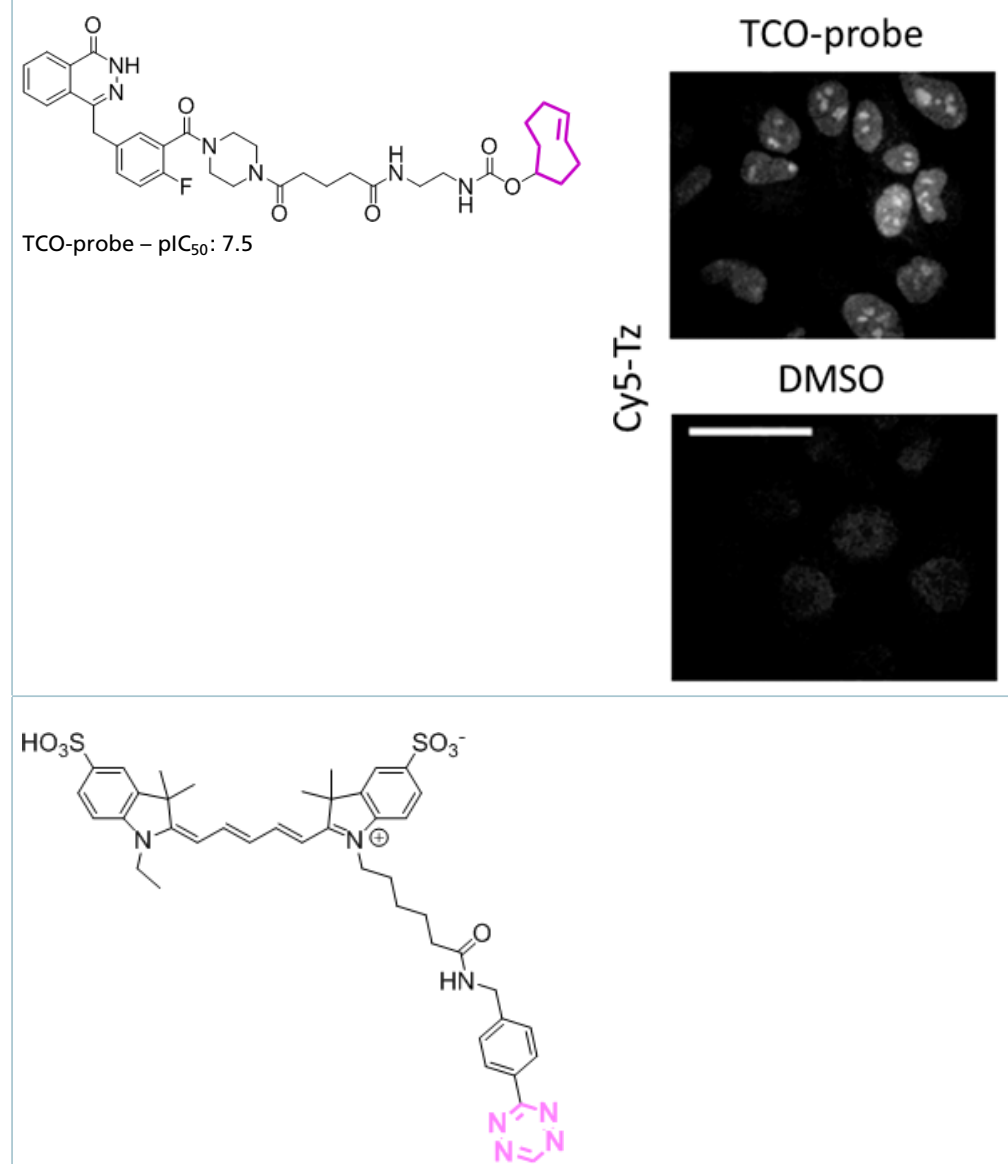

Cy5-Tetrazine

IEDDA reaction

Bioorthogonal moieties are shown in magenta for labelled chemical probes and in pink for the reporter partners. Scale bar $=25 \mu \mathrm{m}$

IEDDA: Inverse-electron demand Diels-Alder; SPAAC: Strain-promotedazide-alkyne cycloaddition; TCO: Trans-cyclooctene.

Adapted with permission from [41] (C) American Chemical Society (2016).

nuclear staining was observed compared with the staining of cells treated with DMSO. The colocalization of the trans-cyclooctene (TCO)-functionalized probe with the target protein PARP1 was assessed by confocal microscopy (Figure 5). This showed that the specific nuclear staining observed with TCO-probe/Cy5-Tz system was correlated with the cellular localization of PARP1 staining by anti-PARP1 antibody and Alexa488 conjugated secondary antibody. 


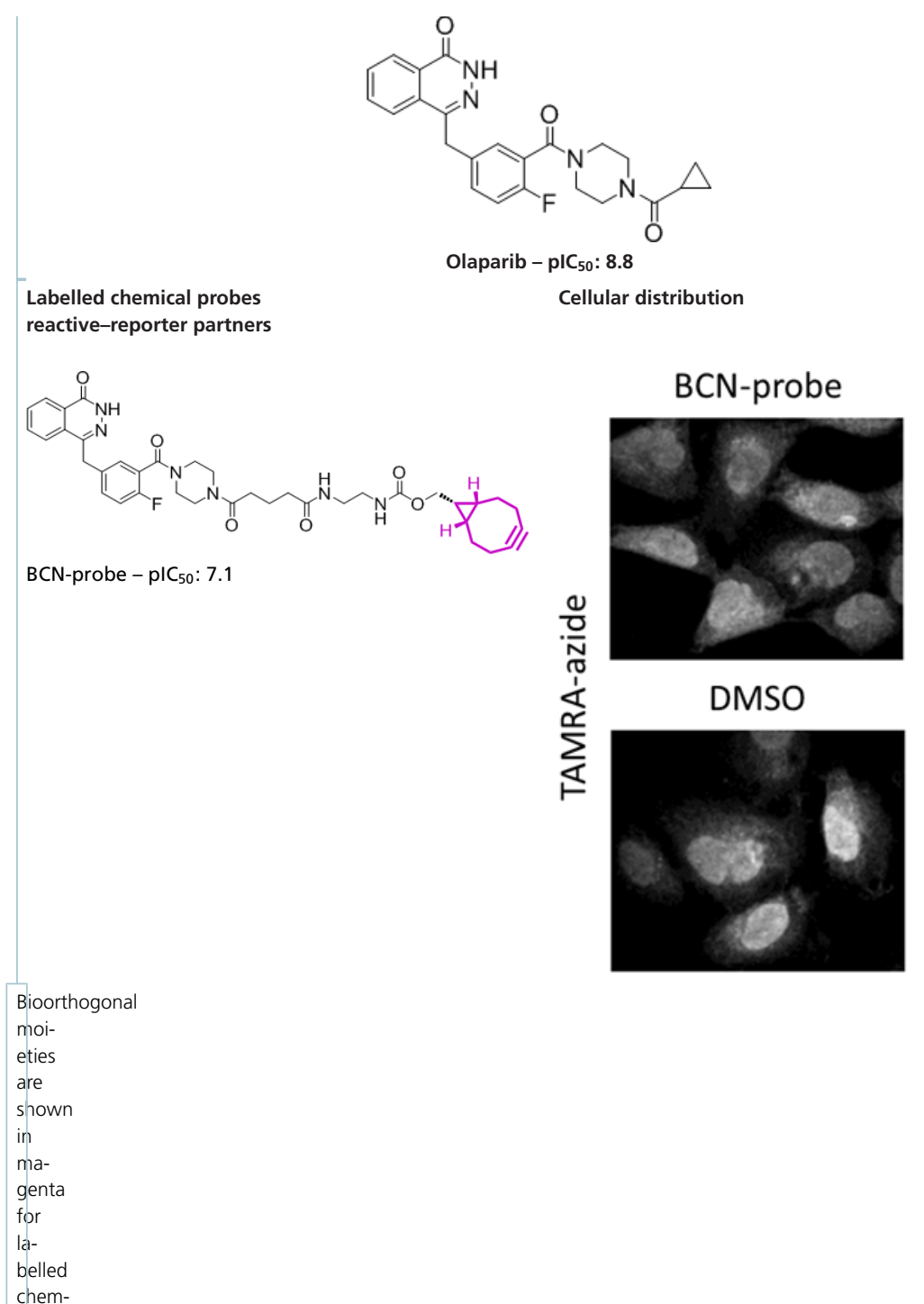



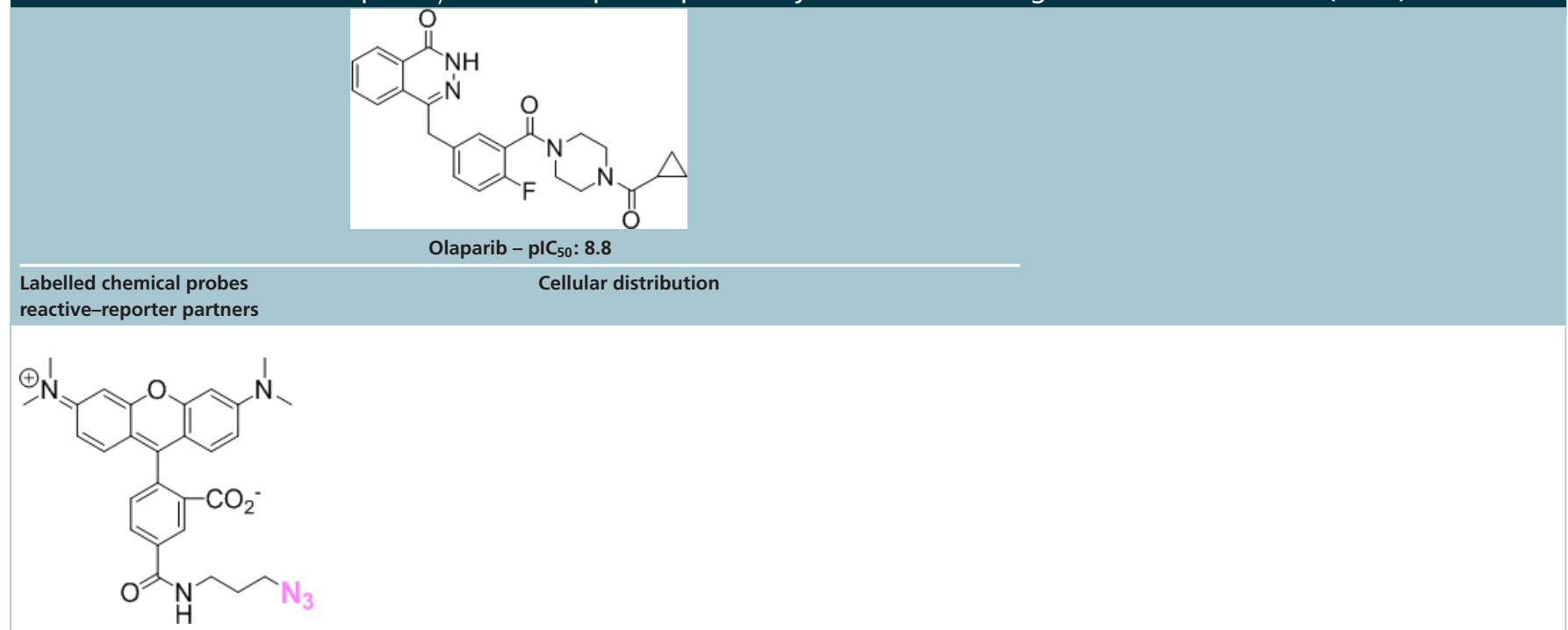

TAMRA-azide

Bioorthogonal moieties are shown in magenta for labelled chemical probes and in pink for the reporter partners. Scale bar $=25 \mu \mathrm{m}$.

IEDDA: Inverse-electron demand Diels-Alder; SPAAC: Strain-promotedazide-alkyne cycloaddition; TCO: Trans-cyclooctene.

Adapted with permission from [41] (C) American Chemical Society (2016).
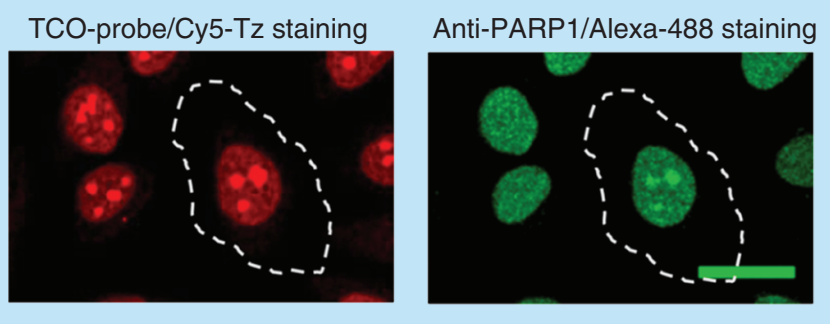

Figure 5. Colocalization of trans-cyclooctene-probe - Cy5-tetraxine conjugate (red) with target protein PARP1 (green). Scale bar $=25 \mu \mathrm{m}$.

Adapted with permission from [41] (c) American Chemical Society (2016).

Similar approaches have been applied to modification of the bioactive molecules MLN8054 [42], BI2536 [43] and PF04217903 [44] to develop labelled chemical probes for assessing their colocalization in live cells with Aurora kinase A, Polo-like kinase 1 and hepatocyte growth factor receptor MET, respectively.

\section{General workflow for measuring target occupancy in cells}

The probe-target colocalization protocol can be adapted to a competition assay format with unmodified ligands in order to determine target occupancy in live cells. The general workflow for determining target occupancy in cells with the bioorthogonal ligation strategy through a competition format is summarized in Figure 6.

Cells are treated with one concentration of the labelled chemical probe followed by a concentration range of the unlabelled ligand.

- For the affinity reporter analysis (upper panel, Figure 6): after cell lysis, bioorthogonal reaction is performed with an affinity reporter coupled to streptavidin beads and bound proteins are analyzed by immunoblotting. Quantification of the level of protein pulled down by the labelled chemical probe shows a dose-dependent 


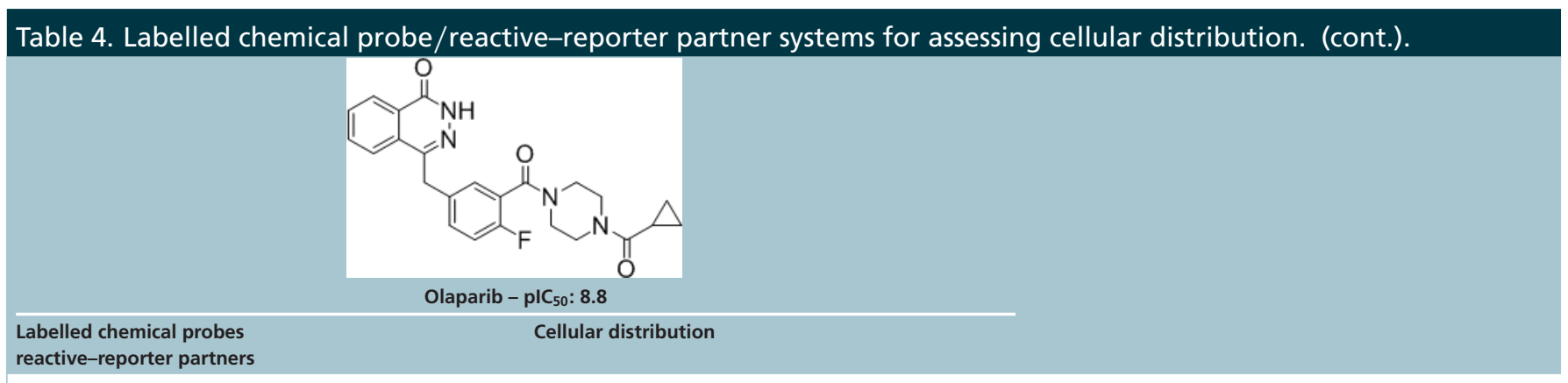

reactive-reporter partners<smiles>C#CC(=O)NCCNC(=O)CCCC(=O)N1CCN(C(=O)c2cc(Cc3n[nH]c(=O)c4ccccc34)ccc2F)CC1</smiles>

Alykne-probe - $\mathrm{plC}_{50}: 8.9$

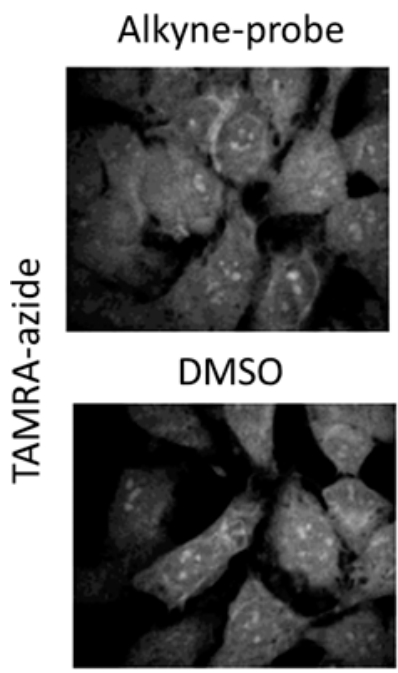<smiles>CN(C)c1ccc2c(-c3ccc(C(=O)NCCCN)cc3C(=O)[O-])c3ccc(=[N+](C)C)cc-3oc2c1</smiles>

TAMRA-azide CUAAC reaction

Bioorthogonal moieties are shown in magenta for labelled chemical probes and in pink for the reporter partners. Scale bar $=25 \mu \mathrm{m}$. IEDDA: Inverse-electron demand Diels-Alder; SPAAC: Strain-promotedazide-alkyne cycloaddition; TCO: Trans-cyclooctene.

Adapted with permission from [41] (C) American Chemical Society (2016).

decrease in protein enrichment with increasing concentration of the ligand, allowing derivation of the cellular binding affinity of the ligand;

- For the imaging reporter analysis (lower panel; Figure 6): the bioorthogonal reaction is performed with an imaging reporter either in live or fixed and permeabilized cells and fluorescently labelled protein-probe complex is detected by confocal microscopy. Quantification of the fluorescence intensity reveals a dose-dependent reduction in fluorescence signal with increasing concentration of the ligand leading to a value for the cellular binding affinity of the ligand.

\section{Recent examples}

The first example highlighted here was a continuation of the study previously described for the cellular colocalization of a TCO-functionalized probe with PARP1 [41]. After characterizing the cellular distribution of the TCO-probe and assessing the colocalization of the TCO-probe with PARP1, two orthogonal reactive-reporter partners - a 


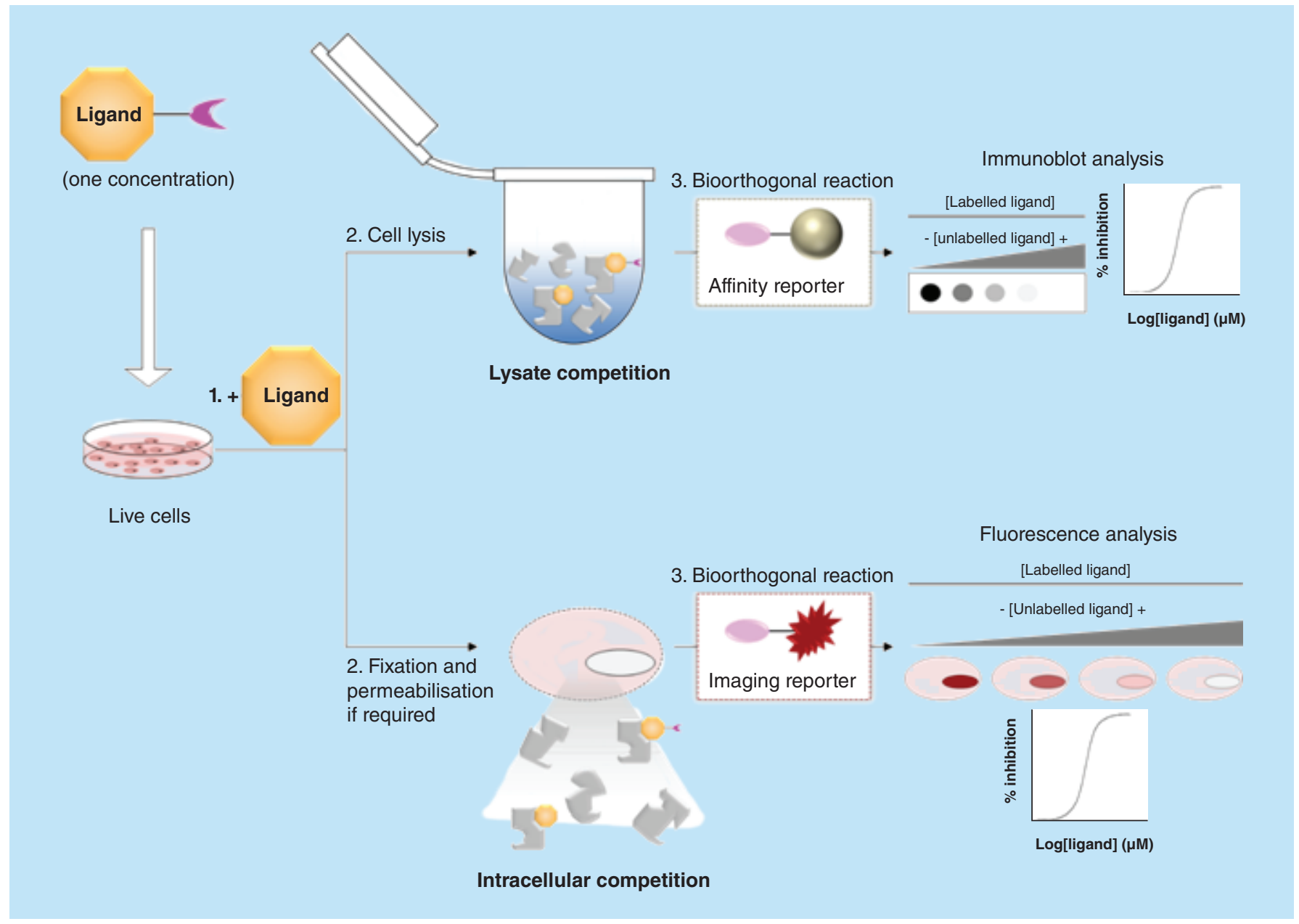

Figure 6. General workflow for measuring target occupancy in cells through the bioorthogonal ligation strategy. See Table 1 for key to symbols.

tetrazine-biotin as an affinity reporter and a tetrazine-fluorophore as an imaging reporter - were used to measure PARP1 occupancy in cells (Table 5).

After optimizing the incubation time, the temperature and the probe concentration in cell extracts, the TCOprobe was used in an affinity enrichment experiment in HeLa cells with biotin-tetrazine as an affinity reporter. Cells were treated with $1 \mu \mathrm{M}$ of TCO-probe and different concentrations of olaparib (0-400 nM). Immunoblotting analysis followed by quantitative mass spectrometry gave an olaparib dose-dependent reduction in target binding of the TCO-probe $\left(\mathrm{pIC}_{50}=8.8\right)$.

In order to further characterize PARP1 engagement with olaparib in cells, the authors applied an orthogonal reactive-reporter partner. Based on the probe-target colocalization experiment, Cy5-tetrazine was selected as an imaging reporter. Similarly to the enrichment assay, cells were treated with $1 \mu \mathrm{M}$ of the TCO-probe and a range of concentrations $(0-250 \mathrm{nM})$ of olaparib. A concentration-dependent decrease of the fluorescent signal was observed in cells by confocal microscopy. Quantification of the fluorescence signal in several nuclei was combined to measure the affinity $\left(\mathrm{pIC}_{50}=9.2\right)$. The same TCO-probe was used to measure the target occupancy of two other PARP1 inhibitors having distinct scaffolds, rucaparib and PJ34. Interestingly, the cellular binding of these inhibitors (rucaparib, $\mathrm{pIC}_{50}=8.8 ; \mathrm{PJ} 34, \mathrm{pIC}_{50}=7.0$ ) were lower than that for olaparib, which correlated with the potencies previously obtained in cell lysates. These results highlight the potential for target occupancy assays with the same labelled chemical probe to rank and triage distinct inhibitors.

Finally in this example, FACS was used to measure target occupancy in single cells. Although this technology does not give subcellular resolution, it has the advantage of high-throughput sorting of a cell population receiving 

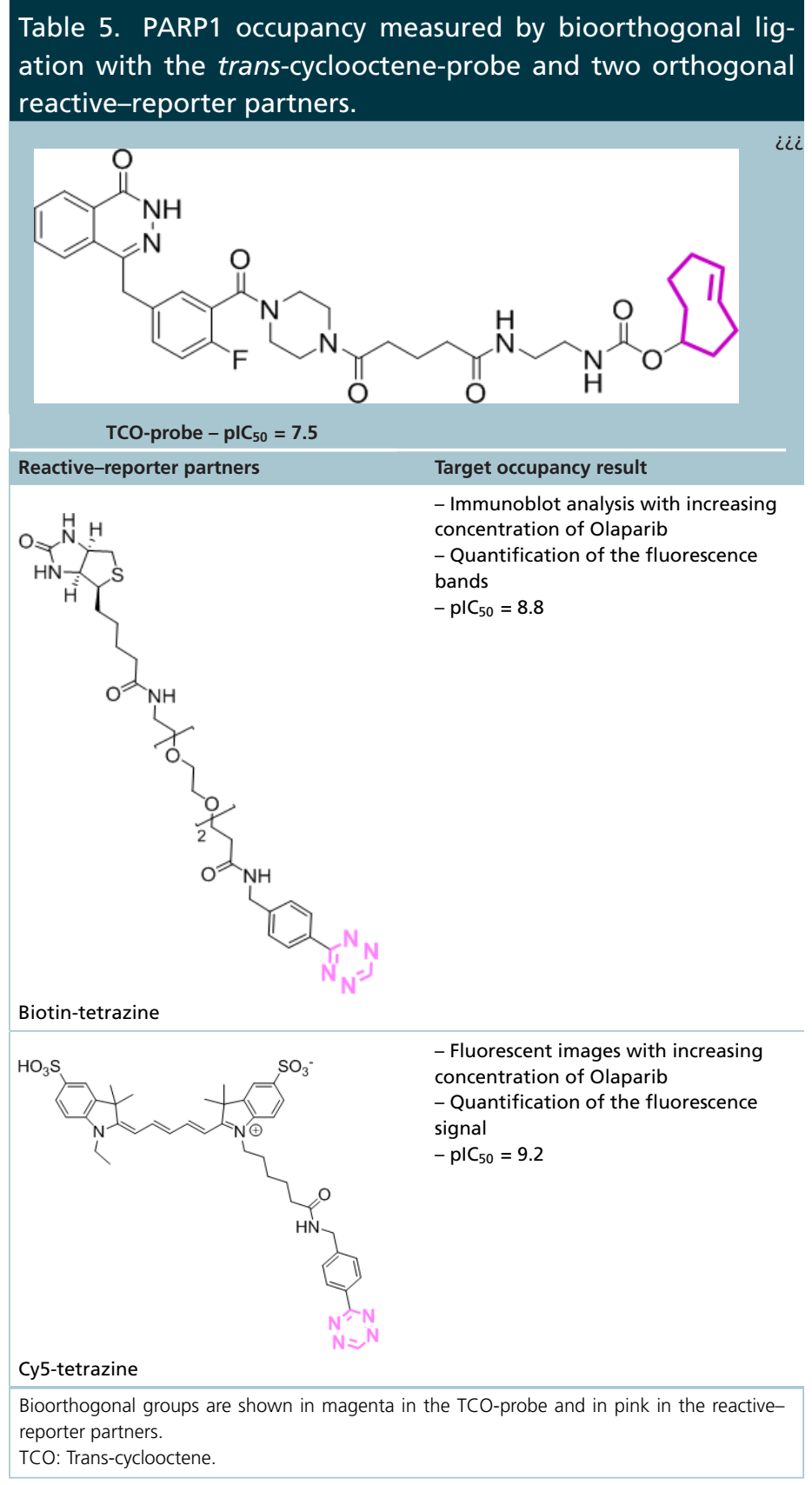

a particular treatment. Jurkat cells were incubated with $1 \mu \mathrm{M}$ of the TCO-probe and different concentrations of olaparib (0-250 nM). After fixation and permeabilization, cells were treated with the Cy5-tetrazine imaging reporter and subjected to flow cytometric analysis. The quantification of the number of fluorescent cells gave binding measurements for olaparib, rucaparib and $\mathrm{PJ} 34$ ( $\mathrm{pIC}_{50}=8.8,8.0$ and 7.0, respectively). These values were in agreement with those obtained with confocal microscopy analysis in previous experiments.

The small differences in the estimated cellular binding affinity of olaparib through these three competitive experiments using a similar probe, but distinct orthogonal reactive-reporter partners highlights the robustness of using labelled chemical probes bearing a bioorthogonal group to assess target occupancy. Bioorthogonal ligation strategies have been successfully applied for other target proteins as summarized in Table 6 .

Inhibitors of the MDM2-p53 protein-protein interaction were studied in a disease-relevant cellular model [45]. The subcellular distributions and co-localization of a set of probes at different concentrations $(0-16 \mu \mathrm{M})$ with the MDM2 target were assessed using different imaging reporters in fixed and live cells. No significant difference was 
Target

Inhibitor labelled chemical probe

MDM2

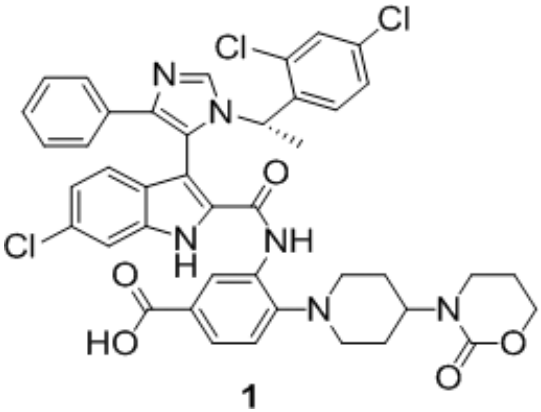

Reactive-reporter partner target occupancy assessment

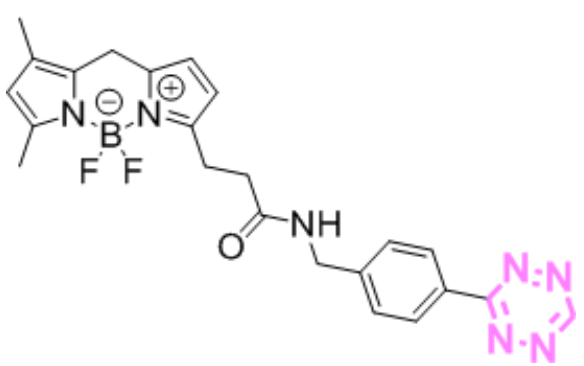

BODIPY-Fl-tetrazine<smiles>C[C@H](c1ccc(Cl)cc1Cl)n1cnc(-c2ccccc2)c1-c1c(C(=O)Nc2cc(C(=O)NCCNC(=O)OC3CCC=CCC3)ccc2N2CCC(N3CCCOC3=O)CC2)[nH]c2cc(Cl)ccc12</smiles> 
Table 6. Recent examples of cellular target occupancy measured using bioorthogonal ligation. (cont.).

Target Inhibitor labelled chemical probe

Reactive-reporter partner target occupancy assessment

BRD4<smiles>CC1=C(C)C2C(c3ccc(Cl)cc3)=N[C@@H](CC(=O)OC(C)(C)C)c3nnc(C)n3[C@H]2S1</smiles><smiles>CC(C)(C)[C@H]1C[C@@H]2NC(=O)N[C@H]2[C@H]1CCCCC(=O)NCCOCCOCCOCCN</smiles>

CUAAC reaction with JQ1-PA<smiles></smiles><smiles>CC1=C(C)C2C(c3ccc(Cl)cc3)=N[C@@H](CC(=O)NCCCNC(=O)OC3CC=CCCC3)c3nnc(C)n3C2S1</smiles> 
observed for the fluorescent staining between fixed and live cells. A clear correlation between MDM2 expression and probe-imaging reporter intensity in the nucleus was observed. In parallel, it was shown that all probes tested were able to induce increased expression of MDM2 in SJSA-1 cells to different extents. The induction of expression of MDM2 protein is a consequence of a feedback mechanism through the $\mathrm{p} 53$ pathway in response to MDM2 inhibition. A probe bearing a TCO group was selected to establish a cellular target occupancy assay in 384-well plates to identify and rank inhibitors in high throughput. Using high content automated imaging analysis at single cell resolution, the cellular binding of several inhibitors was measured. This advanced feature opens the door to the introduction of labelled chemical probe strategies earlier in drug discovery programs as a way to triage compounds based on their capacity to permeate through the cell membrane and reach their intended target in cells.

A TCO-probe, RG7388-TCO, was also developed to study the MDM2-p53 protein-protein interaction [46]. RG7388-TCO induced increased expression of MDM2 in SJSA-1 cells in a dose-dependent manner. To determine the cellular localization of probe-bound MDM2, the turn-on fluorogenic dye, BODIPY-tetrazine, was used as an imaging reporter with enhanced signal-to-background ratio. Nuclear MDM2 staining was colocalized with bound RG7388-TCO - BODIPY-tetrazine conjugate in two MDM2 overexpressing cell lines, SJSA-1 and T778. In a competition assay format where cells were treated with $100 \mathrm{nM}$ of RG7388-TCO for $1 \mathrm{~h}$ followed by incubation for $3 \mathrm{~h}$ with different concentrations (100-1000 nM) of RG7388 before adding the BODIPY-tetrazine, a reduction in the fluorescence signal with increasing concentrations of RG7388 was seen. A 50\% reduction in the fluorescence signal was induced with $100 \mathrm{nM}$ of RG7388 when compared to the negative control (cells receiving DMSO instead of RG7388). Reversing the order of addition of the reagents and treating cells with the same concentration range of RG7388 for $1 \mathrm{~h}$ followed by incubation with $100 \mathrm{nM}$ of RG7388-TCO for $3 \mathrm{~h}$ and subsequently addition of BODIPY-tetrazine also showed a dose-dependent decrease in fluorescence signal, but with a reduction in the magnitude of the inhibition. The difference between these protocols highlighted the incubation time- and concentration-dependency of MDM2 induction following the inhibition of the MDM2-p53 interaction. The total incubation time with $100 \mathrm{nM}$ of RG7388-TCO in the first sequence was $4 \mathrm{~h}$ whereas this time was reduced to $3 \mathrm{~h}$ in the reverse protocol. Therefore, without RG7388 treatment, a longer incubation time with RG7388-TCO would induce expression of a larger amount of MDM2 protein, resulting in an increase in the fluorescence signal. This study highlights the potential for labelled chemical probes to perturb the expression of some target proteins in cells, rendering the quantification of the cellular target occupancy sensitive to the assay protocol. Although the MDM2-p53 protein complex is a particular example, it suggests an important consideration for experimental designs using labelled chemical probes in order to have the highest confidence in the results obtained.

In another example, two labelled chemical probes bearing distinct bioorthogonal groups were used to study the BRD4 belonging to the family of bromodomain and extraterminal domain (BET) proteins [47]. The two probes, named JQ1-PA and JQ1-TCO, were biologically equivalent to the parent molecule JQ1 which was used as the starting point. $2 \mu \mathrm{M}$ of each probe was combined with an affinity reporter in an enrichment experiment to identify genes that were up- and down-regulated upon BET inhibition in different cell lines. Distinct binding modes of BRD4 at genes that responded to BET inhibition compared to those that did not was suggested to account for the differential effects on gene expression. An imaging reporter was used to perform fluorescence microscopy and flow cytometry analysis in cultured cells. This demonstrated a clear colocalization of the probes with BRD4 protein in cell nuclei. Using the FACS strategy, the team revealed an increase in the fluorescent cell population when the cultured cells were treated with increasing concentrations of the labelled chemical probes (from $70 \mathrm{nM}$ to $1 \mu \mathrm{M}$ ). As expected, a decrease in the fluorescent cell population was then observed when cells were further incubated with the unlabelled BET inhibitor, JQ1. This study demonstrates the potential to combine target localization and occupancy assays with gene expression analysis as a downstream biomarker of target engagement.

This approach involving labelled chemical probes bearing bioorthogonal reactive groups has been successfully applied to assess intracellular target-probe colocalization, demonstrate direct target engagement and measure target occupancy in cells. However, the success of such strategies relies on the exposure of the target to the probe, which is highly dependent on the efficiency of the bioorthogonal reaction. Furthermore, this technology has been mainly focused on cellular models, where high resolution is necessary and achievable, whereas PET imaging (discussed below) can provide assessments of target binding in tissues and whole organisms.

\section{Target occupancy in cells with a bioorthogonal reaction-free system}

In the foregoing examples, bioorthogonal reactions were a key element in the target engagement experiments. However, this element is not always required. A new approach using a labelled chemical probe bearing a cell- 


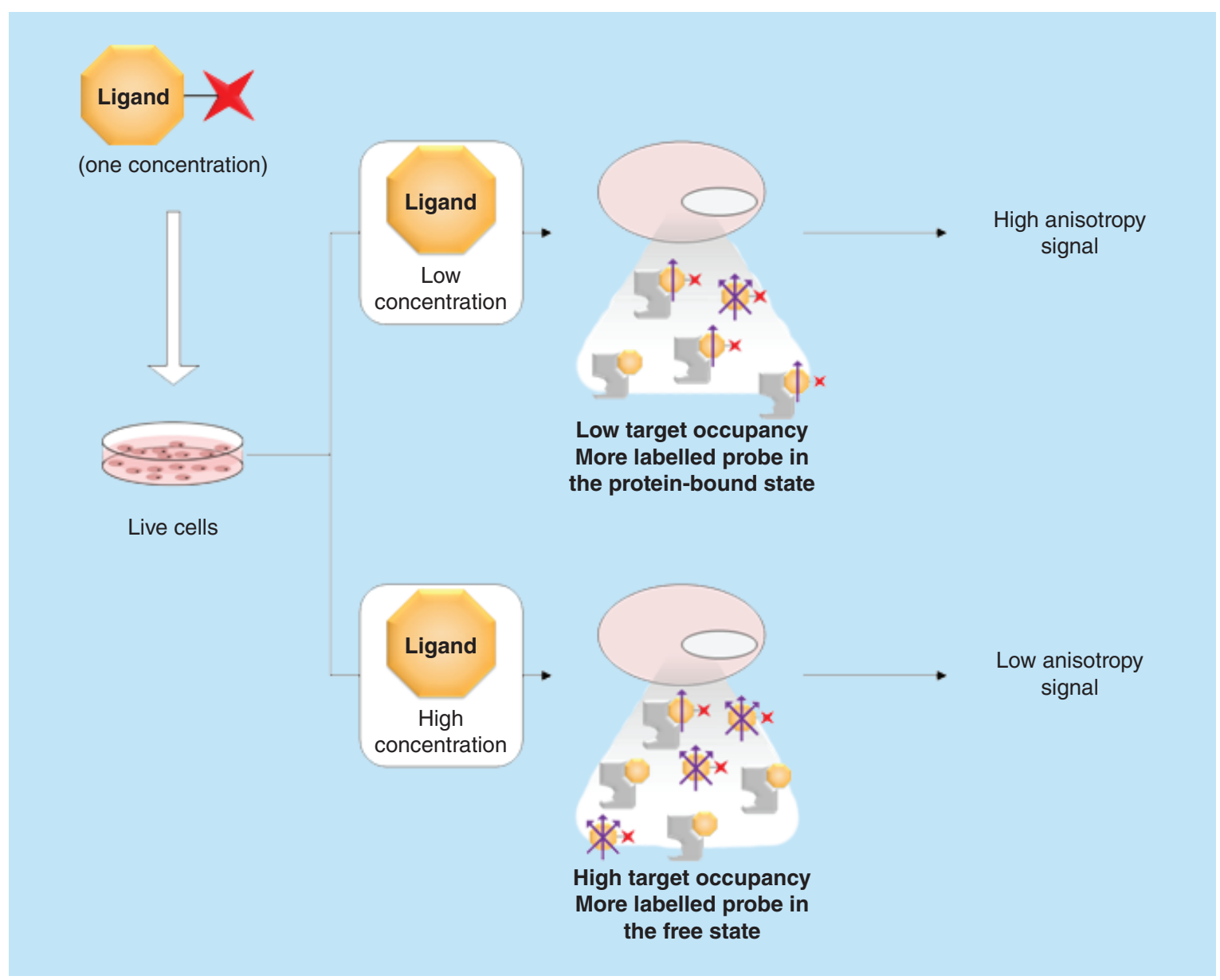

Figure 7. Experimental workflow for measuring target occupancy in cells with a bioorthogonal reaction-free strategy based on detection of fluorescence polarization. See Table 1 for key to symbols.

permeable dye has been recently described [29]. The workflow for measuring target occupancy in cells without using any bioorthogonal reaction is summarized in Figure 7. This strategy relies on fluorescence polarization anisotropy, which can differentiate fluorescent small molecules in the free and protein-bound states. Following polarized light excitation, small molecules - which are bound to a protein - have a slow rotation allowing them to keep their orientation and emit light retaining the incident polarization. Conversely, small molecules in solution have more freedom and rotate quickly, resulting in a depolarization of the emitted light and thus lower polarization anisotropy. Cells are treated with one concentration of the labelled chemical probe and a concentration range of the unlabelled ligand. Quantification of the fluorescence polarization anisotropy signal reveals dose dependent target engagement, with lower anisotropy signal detected when a higher concentration of the unlabelled ligand is present.

In the recent paper [29], an irreversible covalent binding drug, ibrutinib and a reversible binding drug, olaparib, were studied. For clarity of comparison with other methods (section 3 and 4), we focus here on the results obtained with olaparib. This PARP1 inhibitor was functionalized with the cell-permeable fluorophore BODIPY to give the labelled chemical probe OlBFL (Table 7). As for the bioorthogonal moieties described earlier, the fluorophore had to be suitable positioned in order not to affect ligand binding to the protein.

In the fluorescence polarization experiment, the anisotropy signal corresponds to an average of all excited labelled chemical probes present in the cells and thus depends on the concentration of the labelled chemical probe. This concern was highlighted by the authors, implying that at higher OlBFL concentrations there is more unbound OlBFL (because all PARP1 binding sites are occupied), leading to a nonspecific decrease in the anisotropy signal despite high target engagement. To overcome this issue, the study authors derived a parameter, $\Delta \mathrm{r} \cdot \mathrm{int}, \mathrm{which}$ corresponds to the concentration of OlBFL bound to PARP1. Using this metric, the team showed that the $\Delta r \cdot$ int 


Target Inhibitor labelled chemical probe
PARP1

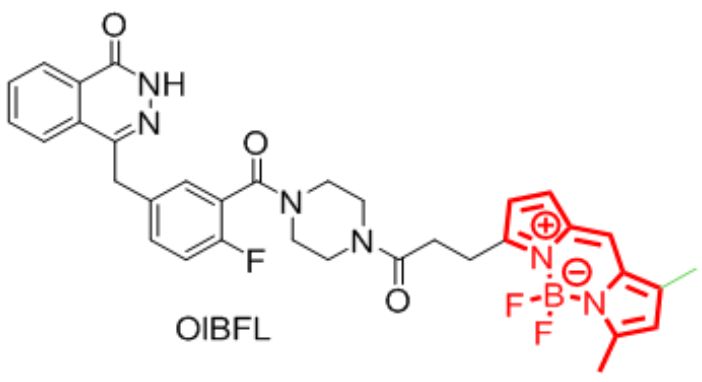

value remained constant when higher concentrations of OlBFL were employed. The cellular localization of OlBFL at one concentration $(500 \mathrm{nM})$ in HT1080 cells was determined and showed a high anisotropy signal in the nucleus where PARP1 is localized. The anisotropy signal was decreased in a dose-dependent manner when cells were exposed to increasing concentrations of olaparib $(0-1 \mu \mathrm{M})$. Through this competition assay, apparent intracellular $\mathrm{k}_{\mathrm{D}}$ values were determined in three cell lines: $1.97 \mathrm{nM}$ in HT1080 (fibrosarcoma), $1.93 \mathrm{nM}$ in HCC1937 (breast cancer) and $1.92 \mathrm{nM}$ in MHHES1 (Ewing's sarcoma).

In summary, the application of a bioorthogonal reaction-free strategy to measure cellular target occupancy is an exciting development. However, due to the necessary size of the appended fluorophore, successful extension to other systems may depend on the size and intrinsic permeability of the parent ligands, as larger molecular weight labelled chemical probes may have limiting cell-permeability. As mentioned previously, the use of PAL chemical probes bearing a photoreactive group and a bioorthogonal group can also be restricted by technical considerations. Hence, at present labelled chemical probes bearing bioorthogonal groups appear to be the most generally applicable strategy to determine target engagement at cellular resolution.

The approaches described so far involved target occupancy studies in cell lines, which are a promising starting point for translating knowledge of biochemical affinity to the in vivo setting. For drug development, the ideal would be to also assess target occupancy in preclinical animal models and ultimately in human patients. In these contexts, PET using radiolabelled chemical probe has received particular attention.

\section{Positron emitting groups as a probe labeling strategy in preclinical models \& clinical settings}

The PET is a clinical imaging tool that has been widely applied to address key questions in drug development and study drug biodistribution and target occupancy [48]. For example, ${ }^{18} \mathrm{~F}$-fluorodeoxyglucose $\left({ }^{18} \mathrm{~F}\right.$-FDG), an ${ }^{18}$ F-labelled glucose derivative, has been extensively used as a PET biomarker to assess the function of metabolic pathways and diagnose or study cancer [49] and neurodegenerative diseases [50,51]. These PET biomarker probes aim to study biological processes in abnormal disease states and also potential changes in response to a therapeutic [52-54]. Here, we focus on the strategy that relies on the functionalization of a bioactive molecule with a radiolabelled atom. Such radiolabelled chemical probes, also called radiotracers, have to display a relatively short half-life in order to allow administration at doses high enough to afford a suitable imaging signal without risking patient health through 
long exposure to ionizing radiation [55]. Due to their short half-lives, ${ }^{11} \mathrm{C}$ (about $20 \mathrm{~min}$ ) and ${ }^{18} \mathrm{~F}$ (about $110 \mathrm{~min}$ ) are the two most frequently employed radiolabelled atoms.

Typically, the radiolabelled chemical probe is administered in vivo at very low doses. Unlike the techniques involving labelled chemical probes previously described, the PET technology provides useful information on the biodistribution of radiolabelled chemical probes in tissues but cannot give resolution at the cellular or intracellular scale. Unlabelled ligand (so-called cold ligand) which competes with the radiotracer can be codosed. Quantitative modulation of the PET signal following the cold ligand administration over a range of doses allows the measurement of target occupancy as a function of ligand concentration in the plasma, leading to the in vivo target affinity of the cold drug. Moreover, this in vivo plasma concentration-target occupancy study facilitates an estimation of the therapeutic index and can guide dose selection for clinical trials [56].

\section{PET methods for preclinical biodistribution \& target engagement studies in vivo}

To allow an easy comparison with the in vitro methods discussed above, the following section focuses on the PET method described for the olaparib-PARP1 drug-protein complex. A recent review has covered PARP1 imaging studies using PET with several radiolabelled chemical probes [57]. Among the olaparib-based PET labelled probes, $\left[{ }^{18} \mathrm{~F}\right]$ PARPi has been studied in mouse models of glioblastoma [58] and small-cell lung cancer (Table 8) [59].

In one study, $\left[{ }^{18} \mathrm{~F}\right]$ PARPi radioactivity was quantified in several organs $2 \mathrm{~h}$ after dosing to a mouse model of glioblastoma [58]. This biodistribution study showed tumor uptake (1.82\% injected dose/g [\%ID/g]), and also that the tumor-to-muscle ratio was 5.1, whereas the tumor-to-brain ratio was 54.9. The authors showed that preinjecting the cold drug olaparib at higher dose reduced the tumor uptake by $87 \%$. Furthermore, significant tracer uptake was also identified in the gastrointestinal tract and hepatobiliary system due to the rapid clearance of $\left[{ }^{18} \mathrm{~F}\right] \mathrm{PARPi}$. The PET quantification of uptake into tumors from images acquired $2 \mathrm{~h}$ postinjection of $\left[{ }^{18} \mathrm{~F}\right] \mathrm{PARPi}$ showed that mice receiving the radiolabelled chemical probe had a radioactivity of $2.15 \% \mathrm{ID} / \mathrm{g}$, whereas mice treating with the probe and olaparib had a reduced radioactivity of $0.28 \% \mathrm{ID} / \mathrm{g}$.

A separate study [59] investigated which image sampling time point after IV dosing of $200-300 \mu \mathrm{Ci}$ of $\left[{ }^{18} \mathrm{~F}\right] \mathrm{PARPi}$ to tumor-bearing mice would provide the highest tumor-to-muscle ratio. Imaging at $120 \mathrm{~min}$ postdose appeared to be the most suitable time point, where $0.87 \% \mathrm{ID} / \mathrm{g}$ tumor uptake was observed while the muscle uptake was 0.12 $\% \mathrm{ID} / \mathrm{g}$. PARP1 engagement was explored for two distinct drugs, olaparib and talazoparib, using a competition assay. The IV administration of either olaparib $(0-50 \mathrm{mg} / \mathrm{kg})$ or talazoparib $(0-15 \mathrm{mg} / \mathrm{kg})$ was followed by 200 $300 \mu \mathrm{Ci}$ of $\left[{ }^{18} \mathrm{~F}\right]$ PARPi $30 \mathrm{~min}$ later and PET images were then recorded after $2 \mathrm{~h}$. As expected, both of the drugs were able to block $\left[{ }^{18} \mathrm{~F}\right]$ PARPi binding, but with different efficacies. For example, at $15 \mathrm{mg} / \mathrm{kg}$, talazoparib and olaparib showed 84 and 75\% inhibition of the PET signal, respectively. Subsequently, the on-target residence time of the two drugs was studied by varying the time $(1-48 \mathrm{~h})$ between the injection of the cold drug and the radiolabelled chemical probe. The doses (olaparib, $50 \mathrm{mg} / \mathrm{kg}$; talazoparib, $0.3 \mathrm{mg} / \mathrm{kg}$ ) of the drugs and the dose of the PET probe were kept constant for all the time points evaluated (1, 2, 4, 8, 24 and $48 \mathrm{~h}$ ). By plotting the percentage of $\left[{ }^{18} \mathrm{~F}\right] \mathrm{PARPi}$ signal inhibited for each time point postdrug administration, the time taken to reduce the $\left[{ }^{18} \mathrm{~F}\right]$ PARPi signal in tumor by half was determined as 9.4 and $9.8 \mathrm{~h}$ for olaparib and talazoparib, respectively. Interestingly, although these two drugs displayed differences in affinity and target engagement, their on-target residence times appeared to be similar.

These two case studies highlight the use of a radiolabelled chemical probe, developed from a known drug, to perform a biodistribution study and quantify target engagement of several inhibitors in animal models. Interestingly, the second study showed that this approach is sensitive enough to differentiate partial and complete target engagement. Detection of partial target engagement for a drug candidate with such an approach might usefully guide adjustments of the dose. Finally, the preclinical characterization of the in vivo kinetics of target engagement can also be assessed by this noninvasive approach.

\section{PET methods for clinical biodistribution \& target engagement studies}

Rucaparib is a PARP inhibitor which is US FDA approved. Recently, a first-in-human study has been developed for the rucaparib-based radiolabelled chemical probe named $\left[{ }^{18} \mathrm{~F}\right]$-Fluorthanatrace or $\left[{ }^{18} \mathrm{~F}\right]$-FTT $[60]$ (NCT02469129 - Table 9). The biodistribution of $\left[{ }^{18} \mathrm{~F}\right]$-FTT in healthy volunteers, as well as in patients with cancer, was compared at several time points [60]. The PET imaging revealed high liver uptake, indicating that the primary route of elimination was through the hepatobiliary system. Moreover, one patient with pancreatic carcinoma and one patient with hepatocellular carcinoma showed $\left[{ }^{18} \mathrm{~F}\right]$-FTT tumor uptake compared with the background tissue 
Table 8. Examples of positron emission tomography imaging using [18F]PARPi radiolabelled chemical probes in two mouse models.<smiles>O=C(c1cc(Cc2n[nH]c(=O)c3ccccc23)ccc1F)N1CCN(C(=O)C2CC2)CC1</smiles>

olaparib<smiles>O=C(c1ccc([18F])cc1)N1CCN(C(=O)c2cc(Cc3n[nH]c(=O)c4ccccc34)ccc2F)CC1</smiles>

tion

- As-

sess-

ing potential

correlation

be-

tween

tumor

size

and

$\left[{ }^{18} \mathrm{~F}\right]$ PARPi

tumor

up-

take

- In

vivo

(murine)

whole

body

imag-

ing

with-

out or

with

pre-

treat-

ment

with

two

drugs

(tar-

get

en-

gage-

ment

imag-

ing)

-

Study-

ing

the in

vivo

kinet-

ics of

the

target

gage-

ment

event

for 
Table 9. First-in-human study of the radiolabelled chemical probe $\left[{ }^{18} \mathrm{~F}\right]-\mathrm{FTT}$.

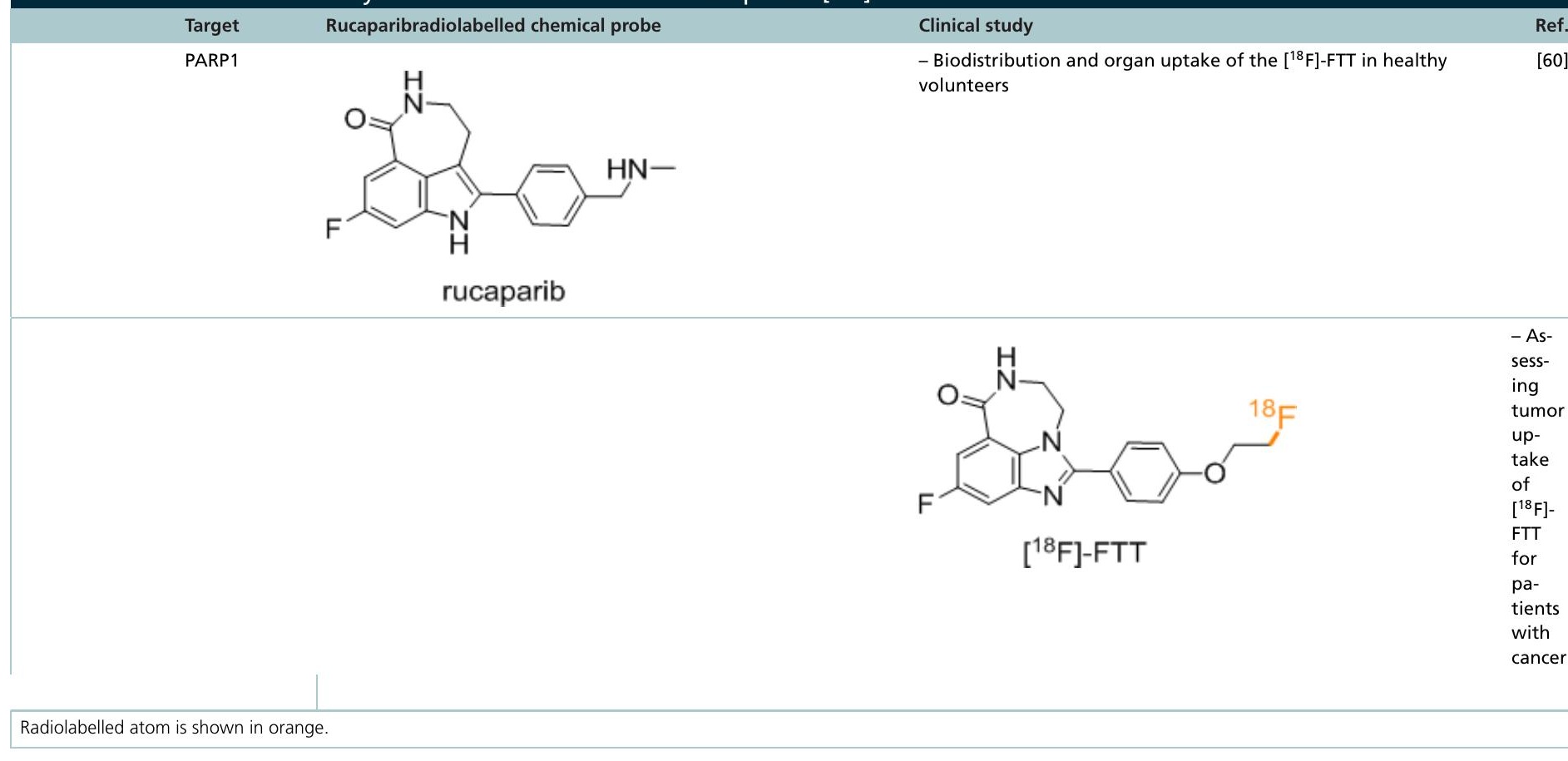

activity. It has been clinically shown that PARP inhibitors may offer treatment for patients with pancreatic cancer who have failed gemcitabine therapy [61], while sensitivity to PARP inhibition has been reported for mouse models of hepatocellular carcinoma [62].

The radiolabelled chemical probe $\left[{ }^{11} \mathrm{C}\right] \mathrm{T}-773$ was studied as a tool to measure PDE10A occupancy by the clinical candidate TAK-063 in healthy subjects (Table 10) [63]. The brain penetration and distribution of [ $\left.{ }^{11} \mathrm{C}\right] \mathrm{T}$ 773 were determined, then the PDE10A occupancy of TAK-063 in relation to its plasma concentration was measured by quantifying the displacement of the radiolabelled chemical probe. For that purpose, TAK-063 was orally administrated and after either 3 or $23 \mathrm{~h},\left[{ }^{11} \mathrm{C}\right] \mathrm{T}-773$ was administered (IV bolus) and PET imaging was performed. Dose dependent target occupancy was seen for TAK-063 after this single dose. At the 3-h time point, the occupancy increased from $2.8 \%$ for a $3 \mathrm{mg}$ dose to $72.1 \%$ for a $1000 \mathrm{mg}$ dose. Furthermore, this increase in PDE10A occupancy was correlated with an increase in TAK-063 plasma concentration.

Similar radiolabelled chemical probes demonstrating drug plasma concentration-dependent target occupancy have been recently described for other central nervous system diseases [64,65].

The PET technology has been widely used to assess the biodistribution of radiolabelled chemical probes in tissues, which is not feasible with the previously discussed fluorescence-labelled chemical probes. Moreover, such radiolabelled chemical probes have been applied with success to evaluate the degree of target occupancy in animal models and in humans. These features display some advantages over the use of labelled chemical probes which are of more applicability to cellular systems, especially when high resolution or information on intracellular location is required. Also, specific facilities are required to carry out radiolabeling synthesis and experiments which can be a limitation of this technology.

\section{Summary}

In summary, this review has highlighted through selected recent examples the use of labelled chemical probes to determine target engagement in living systems. Such efficient and robust chemical tools can provide key data concerning the ligand-protein interaction in a quantifiable manner in cellular models. Through the use of established methodology for PET-radiolabelled chemical probes, this quantitative approach can be extended to animal models and human clinical trials. This is of significance, since there remains a need for the development and wider application of methods to demonstrate on-target occupancy in preclinical drug discovery. 


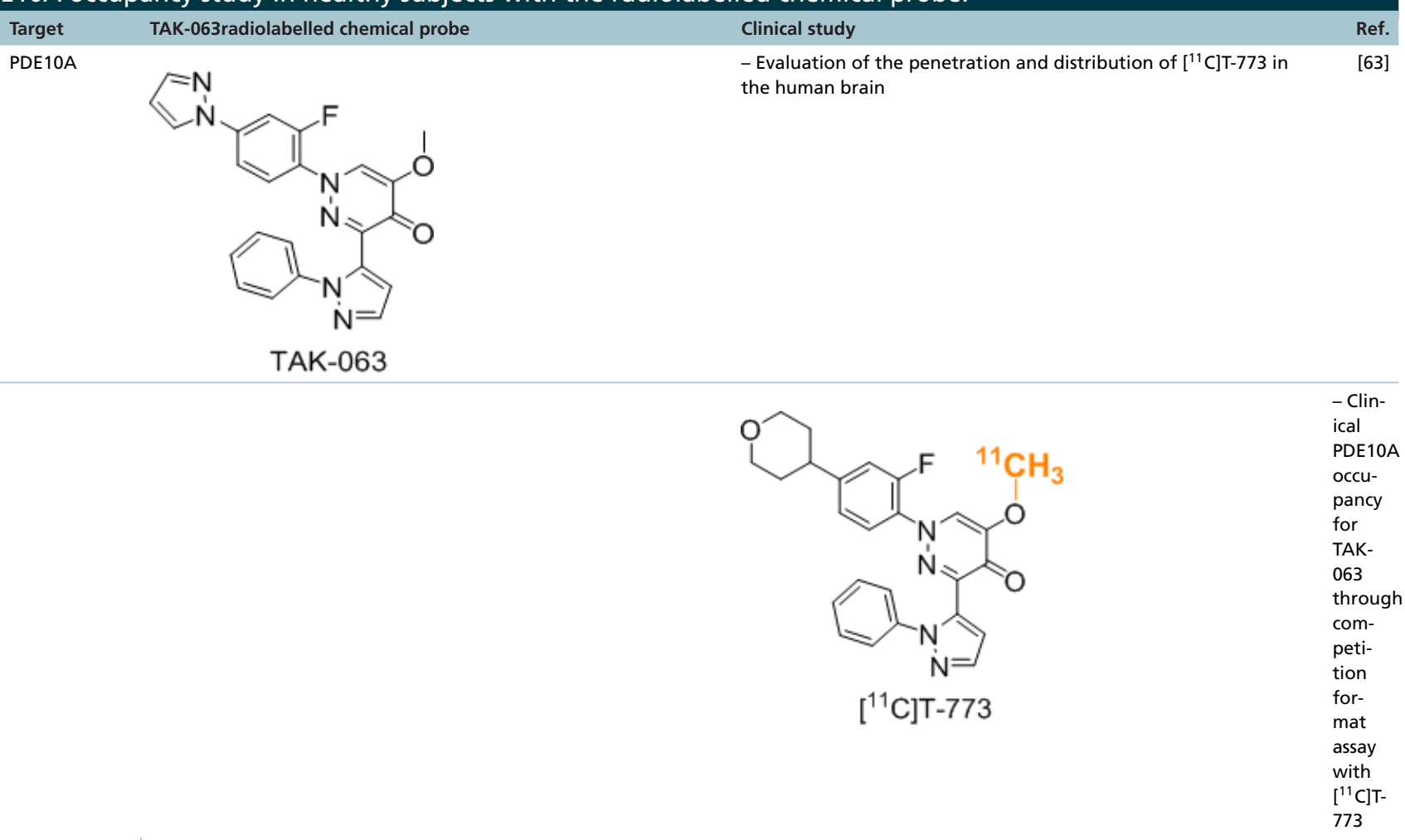

A persuasive example of the potential benefit of introducing target engagement experiments earlier in drug discovery is provided by the example of iniparib, which was initially developed as a PARP inhibitor but failed to show efficacy in a Phase III clinical trial. It was subsequently shown that iniparib did not bind and inhibit PARP at clinically relevant active drug doses [66]. Recently, a comparative study of PARP inhibitors using the labelled chemical probe PARPi-FL (also known as OlBFL) demonstrated the inability of iniparib to block the binding of the probe to PARP in cells, in contrast to a range of other PARP inhibitors including approved clinical agents [59]. Such a result underlines the benefit of using labelled chemical probes in the decision-making process to potentially reduce failures in preclinical, or even clinical, studies due to insufficient interaction of drug candidates to their intended cellular targets.

The multiple potential uses of (radio)-labelled chemical probes is illustrated in Figure 8. labelled chemical probes, developed from suitably potent and selective ligands discovered for a target, can be employed to identify potential off-target effect(s), assess intracellular distribution, measure cellular target occupancy and link the ontarget pharmacology of the ligand to the observed biological effects. Once established, a given labelled chemical probe can be used as a screening tool to evaluate the cellular target occupancy of other ligands binding at the same target site and to prioritize them for further optimization. Radiolabelled chemical probes, particularly $\left[{ }^{18} \mathrm{~F}\right]-\mathrm{PET}$ probes, may be more appropriate for studies in animal models. They can help define ligand biodistribution in animal models, quantify the relationship between target occupancy and plasma concentration to inform on the therapeutic window, and ultimately guide dose selection for clinical trials. Because labelled chemical probes can report at single cell resolution over whole cell populations, whereas radiolabelled chemical probes focus on body compartment resolution, these complementary approaches appear to be adequate and suitable tools for translational studies from cells to animals. 


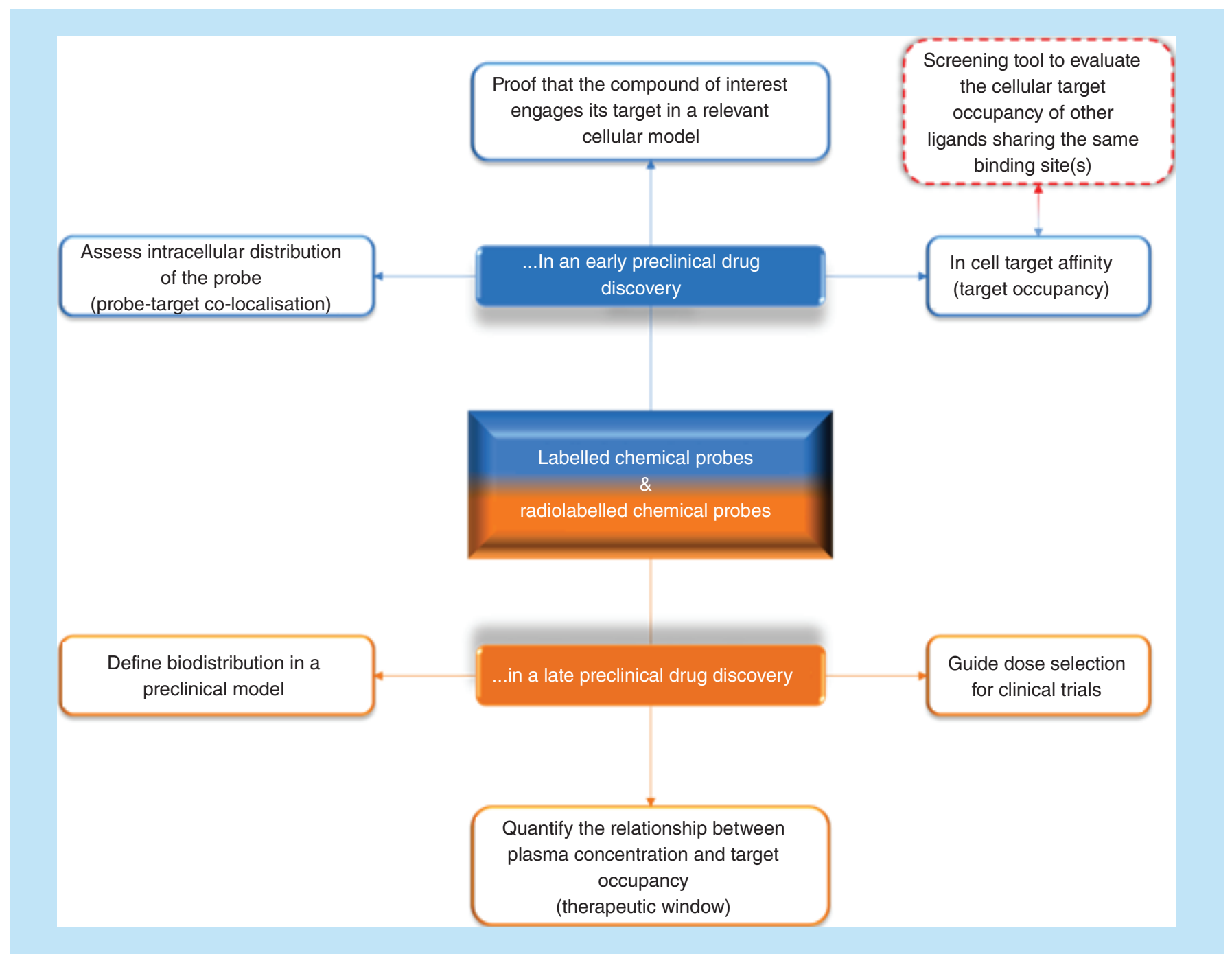

Figure 8. The potential uses of labelled and radiolabelled chemical probes in preclinical drug discovery.

\section{Future prospects}

Transferring labelled chemical probe technologies from cell lines to animal models

As mentioned and highlighted with recent examples throughout sections 3 to 5 , labelled chemical probes have been mainly employed in cell lines to investigate target engagement. However, for translational research, there is a real need to transfer these technologies from the cellular context to more sophisticated animal models. While radiolabelled chemical probes associated with PET methodologies have been successfully applied to animal models, these typically require specialist facilities in terms of radiochemistry, radioimaging and analysis that can limit the accessibility of the approach.

Among the various nonradioactive labelled chemical probes described in this review, two have been studied in vivo; namely, the labelled chemical probe JQ1-TCO bearing a bioorthogonal group [47] and the labelled chemical probe OlBFL functionalized with the cell-permeable fluorophore BODIPY [29]. For JQ1-TCO [47], in vivo administration followed by ex vivo bioorthogonal reaction and FACS analysis enabled the probe distribution across different tissues between healthy mice and tumor-bearing mice to be studied. For OlBFL [29], dorsal window chambers and intravital microscopy, coupled with local administration of the drug, enabled target occupancy to be studied in vivo. However, these rely on ex vivo analysis or require particular surgical techniques which may limit applicability and further advances in this area are still required. 


\section{Raman \& fluorescence spectroscopy}

Alternatively to the (radio)-labelled chemical probes, Raman spectroscopy, which observes the specific vibrational and rotational modes characteristic of a given small molecule, has been applied recently as a label-free imaging method to study the distribution and metabolism pathway of the tyrosine kinase inhibitor Neratinib in cancer cells [67]. As a result, applying this strategy to determine target engagement in cells is potentially feasible and should be observed in the near future.

In addition to this emergent technology, fluorescence spectroscopy involving the use of fluorescent probes has received a considerable interest in medical imaging and diagnosis [68]. Probes with a UV absorption/emission profile in the near-infrared (NIR) allowing tissue penetration have been particularly attractive to study biological processes occurring in animal models. Engineered NIR fluorescent probes have been recently used as imaging biomarkers to monitor changes related to the balance of reactive oxygen species during injuries in living cells and animal models [69-71]. In a similar way, another engineered NIR fluorescent probe derived from the EGF inhibitor gefitinib has been used as theranostic biomarker agent in lung cancer therapy [72]. These NIR fluorescent probes are useful tools allowing for diagnosis and treatment efficacy monitoring in animal models and should be employed more often in the near future as a complementary tool to the (radio)-labelled chemical probes in drug discovery programs.

\section{Impact of protein degradation strategies on determining target engagement}

Strategies for the pharmacological control of protein degradation by modulating the ubiquitin-proteasome system have become of great current interest [73]. One exciting protein degradation methodology uses heterobifunctional molecules (of which the proteolysis targeting chimeras [PROTACs] are the most well known) that contain separate affinity groups for an E3-ubiquitin ligase and a target protein of interest and can trigger the degradation of the protein of interest through its ubiquitination. The interaction between the target protein and the heterobifunctional molecule induces spatial proximity of the target with the E3 ligase complex, allowing poly-ubiquitination of the protein and thereby degradation of the protein via the proteasome.

This approach has been recently used to investigate the in-cell target engagement of a pirin inhibitor [74]. A heterobifunctional molecule was constructed containing the pirin-binding ligand and a thalidomide analog to recruit the cullin4-cereblon E3 ligase complex. The heterobifunctional molecule showed a depletion of the pirin protein in a dose dependent manner. Pirin expression was rescued when cells were pretreated with an unmodified pirin inhibitor prior to adding the probe. Thus targeted degradation can act as readout for assessment of target engagement in a competition format. The properties of such heterobifunctional molecules might appear to be a potential drawback since they typically exhibit high molecular weight and high polar surface area that may limit cell permeability. However, the effectiveness of bioorthogonal reactions for forming the heterobifunctional molecule inside the cells from smaller, more cell permeable components has recently been demonstrated [75]. Two target proteins, BRD4 and ERK1/2, were assessed through such an approach using so-called CLIPTACs (clickformed proteolysis targeting chimeras). A concentration-dependent degradation of both target proteins in cells was achieved using TCO-based chemical probes for the target proteins and a tetrazine-based cereblon-recruiter as the E3-ubiquitin ligase partner.

By combining approaches using different reporter partners to react in cells with the same primed target protein probe, a platform of orthogonal readouts can be envisaged to build confidence in the target engagement event in cells (Figure 9Figure 9). This idea was realized when the same chemical probe (TCO-probe) was used with an affinity reporter (biotin-tetrazine) and an imaging reporter (Cy5-tetrazine) to study the target engagement of olaparib [41]. Extending this approach to incorporate the CLIPTAC strategy [75] could offer an efficient general platform to investigate target engagement in cells using multiple orthogonal readouts.

As highlighted in Figure 9, a single, well-designed labelled chemical probe bearing a bioorthogonal group can be employed in cells with a range of reactive-reporter partners, such as an affinity reporter for protein enrichment experiments, an imaging reporter for protein visualization, and/or cell sorting experiments and an E3-ubiquitin ligase recruiter for protein depletion experiments. All of these probe-reporter pairs can be adapted to target occupancy assays by cotreating cells with competitive unlabelled ligand(s), allowing the cellular binding parameters of multiple ligands to be measured. 


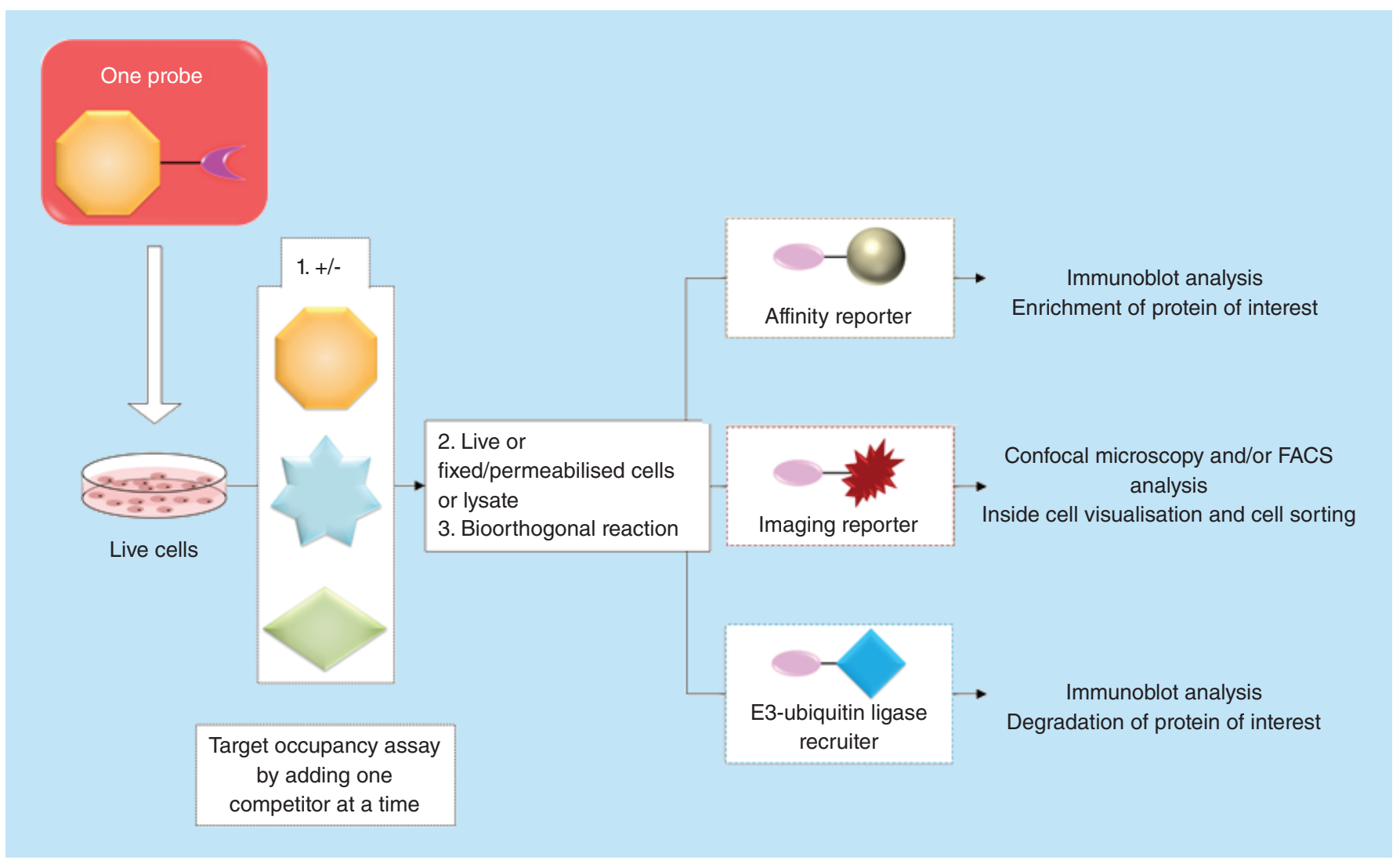

Figure 9. An efficient general platform for parallel investigations of target engagement in cells using multiple approaches.

\section{Future perspective}

Cellular target engagement experiments can answer a central question: does the compound reach and associate with its intended target protein in cells? Target occupancy measurements allow the subsequent question to be addressed: is the association sufficient to account for the observed biological effects? When combined with quantitative approaches for measuring the intracellular bioavailability of a ligand of interest [76], these techniques can provide confidence in the mechanism and specificity of a bioactive molecule, and the tractability of a target pathway for drug development.

Therefore, we can speculate that in a near future (radio)- labelled chemical probes will be used more routinely in both early and late preclinical drug discovery programs. In early preclinical drug discovery, such technologies will be useful not only to assess the direct binding of a ligand to its intended target protein at cellular resolution, but also to compare the in-cell target affinity of ligands having the same target protein to prioritize them for further optimization. In a late preclinical drug discovery, the mechanism of advanced ligands will be confirmed through these technologies which can help in the decision-making process and selection of candidates.

\section{Financial \& competing interests disclosure}

The authors acknowledge funding from Cancer Research UK program (grant number C2739/A22897) and The Institute of Cancer Research. The authors are employees of the Institute of Cancer Research. All authors who are, or have been, employed by The Institute of Cancer Research are subject to a 'Rewards to Inventors Scheme' which may reward contributors to a program that is subsequently licensed. The authors have no other relevant affiliations or financial involvement with any organization or entity with a financial interest in or financial conflict with the subject matter or materials discussed in the manuscript apart from those disclosed.

No writing assistance was utilized in the production of this manuscript. 


\section{Executive summary}

\section{Target engagement}

- Target engagement experiments refer to the study of the direct binding of a ligand to its intended target protein in living systems.

- (Radio)-labelled chemical probes are the key actors to carry out such experiments.

Target engagement experiments that rely on a ligation strategy

- These experiments involve labelled chemical probes and distinct reactive-reporter partners that are assembled together in cells using bioorthogonal ligation strategies.

- Unlabelled ligand is commonly used in combination with the labelled probe to measure target occupancy and cellular binding affinity through a competition assay format.

Target engagement experiments that rely on ligation-free strategies

- Labelled chemical probes functionalized with a cell-permeable dye or radiolabelled chemical probes functionalized with a radiolabelled atom are suitable for target engagement experiments.

- Positron emission tomography imaging of biodistribution and target engagement in tissues using radiolabelled chemical probes is clinically applicable. 


\section{Open access}

This work is licensed under the Creative Commons Attribution 4.0 License. To view a copy of this license, visit http://creativecommons.org/licenses/by-nc-nd/4.0/

\section{References}

Papers of special note have been highlighted as: $\bullet$ of interest; $\bullet \bullet$ of considerable interest

1. Bunnage ME, Chekler EL, Jones LH. Target validation using chemical probes. Nat. Chem. Biol. 9(4), 195-199 (2013).

- Definition of four scientific pillars underlying successful drug discovery programs through robust target validation.

2. Morgan P, Van Der Graaf PH, Arrowsmith J et al. Can the flow of medicines be improved? Fundamental pharmacokinetic and pharmacological principles toward improving Phase II survival. Drug Discov. Today 17(9-10), 419-424 (2012).

3. Hann MM, Simpson GL. Intracellular drug concentration and disposition - the missing link? Methods 68(2), 283-285 (2014).

4. Smyth LA, Collins I. Measuring and interpreting the selectivity of protein kinase inhibitors. J. Chem. Biol. 2(3), 131-151 (2009).

5. Simon GM, Niphakis MJ, Cravatt BF. Determining target engagement in living systems. Nat. Chem. Biol. 9(4), 200-205 (2013).

6. Schürmann M, Janning P, Ziegler S, Waldmann H. Small-molecule target engagement in cells. Cell Chem. Biol. 23(4), 435-441 (2016).

7. Martinez Molina D, Jafari R, Ignatushchenko $\mathrm{M}$ et al. Monitoring drug target engagement in cells and tissues using the cellular thermal shift assay. Science 341(6141), 84-87 (2013).

- Introduction of the label-free cellular thermal shift assay methodology for measuring cellular target engagement.

8. Cimmperman P, Baranauskiene L, Jachimoviciūte $S$ et al. A quantitative model of thermal stabilization and destabilization of proteins by ligands. Biophys. J. 95(7), 3222-3231 (2008).

9. Blagg J, Workman P. Choose and use your chemical probe wisely to explore cancer biology. Cancer Cell 32(1), 9-25 (2017).

10. Frye SV. The art of the chemical probe. Nat. Chem. Biol. 6(3), 159-161 (2010).

11. Workman P, Collins I. Probing the probes: fitness factors for small molecule tools. Chem. Biol. 17(6), 561-577 (2010).

12. Arrowsmith CH, Audia JE, Austin C et al. The promise and peril of chemical probes. Nat. Chem. Biol. 11(8), 536-541 (2015).

- Comprehensive review of the properties of high quality pharmacological probes and description of the Chemical Probes Portal, a resource to support the selection of high quality of pharmacological tools.

13. Antolin AA, Tym JE, Komianou A, Collins I, Workman P, Al-Lazikani B. Objective, quantitative, data-driven assessment of chemical probes. Cell Chem. Biol. 25(2), 194-205.e195 (2018).

14. Lang K, Chin JW. Bioorthogonal reactions for labeling proteins. ACS Chem. Biol. 9(1), 16-20 (2014).

15. Chen X, Wu YW. Selective chemical labeling of proteins. Org. Biomol. Chem. 14, 5417-5439 (2016).

16. Sletten EM, Bertozzi CR. Bioorthogonal chemistry: fishing for selectivity in a sea of functionality. Angew. Chem. Int. Ed. Engl. 48(38), 6974-6998 (2009).

17. Ramil CP, Lin Q. Bioorthogonal chemistry: strategies and recent developments. Chem. Commun. (Camb.) 49(94), 11007-11022 (2013).

18. Shieh P, Bertozzi CR. Design strategies for bioorthogonal smart probes. Org. Biomol. Chem. 12(46), 9307-9320 (2014).

19. Patterson DM, Nazarova LA, Prescher JA. Finding the right (bioorthogonal) chemistry. ACS Chem. Biol. 9(3), 592-605 (2014).

20. Row RD, Prescher JA. Constructing new bioorthogonal reagents and reactions. Acc. Chem. Res. 51(5), 1073-1081 (2018).

21. Wu H, Devaraj NK. Advances in tetrazine bioorthogonal chemistry driven by the synthesis of novel tetrzines and dienophiles. Acc. Chem. Res. 51(5), 1249-1259 (2018).

22. Kang K, Park J, Kim E. Tetrazine ligation for chemical proteomics. Proteome Sci. 15, 15 (2016).

23. McFedries A, Schwaid A, Saghatelian A. Methods for the elucidation of protein-small molecule interactions. Chem. Biol. 20(5), 667-673 (2013).

24. Devaraj NK, Hilderbrand S, Upadhyay R, Mazitschek R, Weissleder R. Bioorthogonal turn-on probes for imaging small molecules inside living cells. Angew. Chem. Int. Ed. Engl. 49(16), 2869-2872 (2010).

-• Discovery and application of fluorogenic turn-on tetrazine-based imaging reporters.

25. Carlson JC, Meimetis LG, Hilderbrand SA, Weissleder R. BODIPY-tetrazine derivatives as superbright bioorthogonal turn-on probes. Angew. Chem. Int. Ed. Engl. 52(27), 6917-6920 (2013).

26. Meimetis LG, Carlson JC, Giedt RJ, Kohler RH, Weissleder R. Ultrafluorogenic coumarin-tetrazine probes for real-time biological imaging. Angew. Chem. Int. Ed. Engl. 53(29), 7531-7534 (2014).

27. Wieczorek A, Werther P, Euchner J, Wombacher R. Green- to far-red-emitting fluorogenic tetrazine probes - synthetic access and no-wash protein imaging inside living cells. Chem. Sci. 8(2), 1506-1510 (2017).

28. Lapinsky DJ. Tandem photoaffinity labeling-bioorthogonal conjugation in medicinal chemistry. Bio. Org. Med. Chem. 20(21), 6237-6247 (2012). 
29. Dubach JM, Kim E, Yang K et al. Quantitating drug-target engagement in single cells in vitro and in vivo. Nat. Chem. Biol. 13(2), 168-173 (2017).

-• Demonstration of PARP1 occupancy measurement with labelled chemical probes bearing a cell-permeable dye.

30. Tanaka Y, Bond MR, Kohler JJ. Photocrosslinkers illuminate interactions in living cells. Mol. Biosyst. 4(6), 473-480 (2008).

31. Geurink PP, Prely LM, Van Der Marel GA, Bischoff R, Overkleeft HS. Photoaffinity labeling in activity-based protein profiling. Top Curr. Chem. 324, 85-113 (2012).

32. Schülke JP, McAllister LA, Geoghegan KF et al. Chemoproteomics demonstrates target engagement and exquisite selectivity of the clinical phosphodiesterase 10A inhibitor MP-10 in its native environment. ACS Chem. Biol. 9(12), 2823-2832 (2014).

33. Crump CJ, Murrey HE, Ballard TE et al. Development of sulfonamide photoaffinity inhibitors for probing cellular $\gamma$-secretase. ACS Chem. Neurosci. 7(8), 1166-1173 (2016).

34. Soethoudt M, Stolze SC, Westphal MV et al. Selective photoaffinity probe that enables assessment of cannabinoid $\mathrm{CB}_{2}$ receptor expression and ligand engagement in human cells. J. Am. Chem. Soc. 140(19), 6067-6075 (2018).

35. Parker CG, Galmozzi A, Wang Y et al. Ligand and target discovery by fragment-based screening in human cells. Cell 168(3), 527-541.e529 (2017).

36. Gertsik N, Am Ende CW, Geoghegan KF et al. Mapping the binding site of BMS-708163 on $\gamma$-secretase with cleavable photoprobes. Cell Chem. Biol. 24(1), 3-8 (2017).

37. Smith E, Collins I. Photoaffinity labeling in target- and binding-site identification. Future Med. Chem. 7(2), 159-183 (2015).

38. Sipthorp J, Lebraud H, Gilley R et al. Visualization of endogenous ERK1/2 in cells with a bioorthogonal covalent probe. Bioconjug. Chem. 28(6), 1677-1683 (2017).

39. Lebraud H, Wright DJ, East CE, Holding FP, O’Reilly M, Heightman TD. In-gel activity-based protein profiling of a clickable covalent ERK1/2 inhibitor. Mol. Biosyst. 12, 2867-2874 (2016).

40. Chen YC, Zhang C. A Chemoproteomic method for identifying cellular targets of covalent kinase inhibitors. Genes Cancer 7(5-6), 148-153 (2016).

41. Rutkowska A, Thomson DW, Vappiani J et al. A modular probe strategy for drug localization, target identification and target occupancy measurement on single cell level. ACS Chem. Biol. 11(9), 2541-2550 (2016).

•. Demonstration of TCO-probe/PARP1 colocalization in cells and PARP1 occupancy measurement using a bioorthogonal ligation strategy.

42. Yang KS, Budin G, Reiner T, Vinegoni C, Weissleder R. Bioorthogonal imaging of aurora kinase A in live cells. Angew. Chem. Int. Ed. Engl. 51(27), 6598-6603 (2012).

43. Budin G, Yang KS, Reiner T, Weissleder R. Bioorthogonal probes for polo-like kinase 1 imaging and quantification. Angew. Chem. Int. Ed. Engl. 50(40), 9378-9381 (2011).

44. Kim E, Yang KS, Weissleder R. Bioorthogonal small molecule imaging agents allow single-cell imaging of MET. PLoS ONE 8(11), e81275 (2013).

45. D’Alessandro PL, Buschmann N, Kaufmann M et al. Bioorthogonal probes for the study of MDM2-p53 inhibitors in cells and development of high-content screening assays for drug discovery. Angew. Chem. Int. Ed. Engl. 55(52), 16026-16030 (2016).

46. Lebraud H, Noble RA, Phillips $\mathrm{N}$ et al. Highly potent clickable probe for cellular imaging of MDM2 and assessing dynamic responses to MDM2-p53 inhibition. Bioconjug. Chem. 29(6), 2100-2106 (2018).

47. Tyler DS, Vappiani J, Cañeque T et al. Click chemistry enables preclinical evaluation of targeted epigenetic therapies. Science 356(6345), 1397-1401 (2017).

-• In vivo administration and ex vivo analysis for target engagement through the bioorthogonal ligation strategy.

48. Matthews PM, Rabiner I, Gunn R. Non-invasive imaging in experimental medicine for drug development. Curr. Opin. Pharmacol. 11(5), 501-507 (2011).

49. Croteau E, Renaud JM, Richard MA, Ruddy TD, Bénard F, Dekemp RA. PET metabolic biomarkers for cancer. Biomark. Cancer 8(Suppl. 2), 61-69 (2016).

50. Nicastro N, Garibotto V, Burkhard PR. The role of molecular imaging in assessing degenerative Parkinsonism - an updated review. Swiss Med. Wkly 148, w14621 (2018).

51. Rice L, Bisdas S. The diagnostic value of FDG and amyloid PET in Alzheimer's disease: a systematic review. Eur. J. Radiol. 94, 16-24 (2017).

52. Sathekge MM, Ankrah AO, Lawal I, Vorster M. Monitoring response to therapy. Semin. Nucl. Med. 48(2), 166-181 (2018).

53. Dupont AC, Largeau B, Guilloteau D, Santiago Ribeiro MJ, Arlicot N. The place of PET to assess new therapeutic effectiveness in neurodegenerative diseases. Contrast Media Mol. Imaging 2018, 7043578 (2018).

54. Knight JC, Koustoulidou S, Cornelissen B. Imaging the DNA damage response with PET and SPECT. Eur. J. Nucl. Med. Mol. Imaging 44(6), 1065-1078 (2017). 
55. Matthews PM, Rabiner EA, Passchier J, Gunn RN. Positron emission tomography molecular imaging for drug development. Br. J. Clin. Pharmacol. 73(2), 175-186 (2012).

56. Hargreaves RJ, Rabiner EA. Translational PET imaging research. Neurobiol. Dis. 61, 32-38 (2014).

- Comprehensive description of the positron emission tomography in vivo imaging tools for target engagement experiments.

57. Carney B, Kossatz S, Reiner T. Molecular Imaging of PARP. J. Nucl. Med. 58(7), 1025-1030 (2017).

58. Carney B, Carlucci G, Salinas B et al. Non-invasive PET imaging of PARP1 expression in glioblastoma models. Mol. Imaging Biol. 18(3), 386-392 (2016).

59. Carney B, Kossatz S, Lok BH et al. Target engagement imaging of PARP inhibitors in small-cell lung cancer. Nat. Commun. $9(1), 176$ (2018).

60. Michel LS, Dyroff S, Brooks FJ et al. PET of poly (ADP-ribose) polymerase activity in cancer: preclinical assessment and first in-human studies. Radiology 282(2), 453-463 (2017).

61. Kaufman B, Shapira-Frommer R, Schmutzler RK et al. Olaparib monotherapy in patients with advanced cancer and a germline BRCA1/2 mutation. J. Clin. Oncol. 33(3), 244-250 (2015).

62. Quiles-Perez R, Muñoz-Gámez JA, Ruiz-Extremera A et al. Inhibition of poly adenosine diphosphate-ribose polymerase decreases hepatocellular carcinoma growth by modulation of tumor-related gene expression. Hepatology 51(1), 255-266 (2010).

63. Takano A, Stenkrona P, Stepanov V et al. A human [(11)C]T-773 PET study of PDE10A binding after oral administration of TAK-063, a PDE10A inhibitor. Neuroimage 141, 10-17 (2016).

64. Naganawa M, Dickinson GL, Zheng MQ et al. Receptor occupancy of the א-opioid antagonist LY2456302 measured with positron emission tomography and the novel radiotracer 11C-LY2795050. J. Pharmacol. Exp. Ther. 356(2), 260-266 (2016).

65. Gallezot JD, Planeta B, Nabulsi N et al. Determination of receptor occupancy in the presence of mass dose. J. Cereb. Blood Flow Metab. 37(3), 1095-1107 (2017).

66. Patel AG, De Lorenzo SB, Flatten KS, Poirier GG, Kaufmann SH. Failure of iniparib to inhibit poly(ADP-ribose) polymerase in vitro. Clin. Cancer Res. 18(6), 1655-1662 (2012).

67. Aljakouch K, Lechtonen T, Yosef HK et al. Raman microspectroscopic evidence for the metabolism of a tyrosine kinase inhibitor, neratinib, in cancer cells. Angew. Chem. Int. Ed. Engl. 57(24), 7250-7254 (2018).

68. Gao M, Yu F, Lv C, Choo J, Chen L. Fluorescent chemical probes for accurate tumor diagnosis and targeting therapy. Chem. Soc. Rev. 46, 2237-2271 (2017).

69. Han X, Wang R, Song X, Yu F, Lv C, Chen L. A mitochondrial-targeting near-infrared fluorescent probe for bioimaging and evaluating endogenous superoxide anion changes during is chemia/reperfusion injury. Biomaterials 156, 134-146 (2018).

70. Gao M, Wang R, Yu F, Chen L. Evaluation of sulfane sulfur bioeffects via a mitochondria-targeting selenium-containing near-infrared fluorescent probe. Biomaterials 160, 1-14 (2018).

71. Wang R, Han X, You J, Yu F, Chen L. Ratiometric near-infrared fluorescent probe for synergetic detection of monoamine oxidase b and its contribution to oxidative stress in cell and mice aging models. Anal. Chem. 90(6), 4054-4061 (2018).

72. Song X, Han X, Yu F, Zhang X, Chen L, Lv C. Polyamine-targeting gefitinib prodrug and ots near-infrared fluorescent theranostic derivative for monitoring drug delivery and lung cancer therapy. Theranostics 8(8), 2217-2228 (2018).

73. Collins I, Wang H, Caldwell JJ, Chopra R. Chemical approaches to targeted protein degradation through modulation of the ubiquitin-proteasome pathway. Bio. Chem. J. 474(7), 1127-1147 (2017).

74. Chessum NEA, Sharp SY, Caldwell JJ et al. Demonstrating in-cell target engagement using a pirin protein degradation probe (CCT367766). J. Med. Chem. 61(3), 918-933 (2018).

75. Lebraud H, Wright DJ, Johnson CN, Heightman TD. Protein degradation by in-cell self-assembly of proteolysis targeting chimeras. ACS Cent. Sci. 2(12), 927-934 (2016).

-• Introduction of the click-formed proteolysis targeting chimera approach for target engagement through the protein degradation strategy.

76. Mateus A, Gordon LJ, Wayne GJ et al. Prediction of intracellular exposure bridges the gap between target- and cell-based drug discovery. Proc. Natl Acad. Sci. USA 114(30), E6231-E6239 (2017). 
